\title{
Transcriptome analysis of the filamentous fungus Aspergillus nidulans directed to the global identification of promoters
}

\author{
Christopher Sibthorp ${ }^{1}$, Huihai Wu², Gwendolyn Cowley ${ }^{1}$, Prudence W H Wong², Paulius Palaima',
} Igor Y Morozov ${ }^{1,3}$, Gareth D Weedall ${ }^{1 *}$ and Mark X Caddick ${ }^{1 *}$

\begin{abstract}
Background: The filamentous fungus Aspergillus nidulans has been a tractable model organism for cell biology and genetics for over 60 years. It is among a large number of Aspergilli whose genomes have been sequenced since 2005, including medically and industrially important species. In order to advance our knowledge of its biology and increase its utility as a genetic model by improving gene annotation we sequenced the transcriptome of A. nidulans with a focus on $5^{\prime}$ end analysis.

Results: Strand-specific whole transcriptome sequencing showed that 80-95\% of annotated genes appear to be expressed across the conditions tested. We estimate that the total gene number should be increased by approximately 1000, to 11,800 . With respect to splicing $8.3 \%$ of genes had multiple alternative transcripts, but alternative splicing by exon-skipping was very rare. $75 \%$ of annotated genes showed some level of antisense transcription and for one gene, meaB, we demonstrated the antisense transcript has a regulatory role. Specific sequencing of the $5^{\prime}$ ends of transcripts was used for genome wide mapping of transcription start sites, allowing us to interrogate over 7000 promoters and $5^{\prime}$ untranslated regions.
\end{abstract}

Conclusions: Our data has revealed the complexity of the A. nidulans transcriptome and contributed to improved genome annotation. The data can be viewed on the AspGD genome browser.

Keywords: Aspergillus nidulans, Transcriptome, RNA-seq, Gene annotation, Alternative splicing, Natural antisense transcripts, Transcription start sites, Transcription factor binding sites

\section{Background}

The filamentous fungus Aspergillus nidulans is a model organism for many aspects of cell biology and genetics. Additionally, the aspergilli themselves include fungi of biomedical, agricultural and industrial significance [1-5]. Thus it is important to extend our understanding of A. nidulans to facilitate analysis of key processes which underpin fungal pathogenicity and biotechnological applications. The $A$. nidulans genome was sequenced and annotated in 2005 [6] and the genome annotation has been updated several times since then. It is among a relatively large number of sequenced Aspergillus genomes, genomic data for which are accessible via a number of public web resources [7-10]. Despite this

\footnotetext{
* Correspondence: gweedall@liv.ac.uk; caddick@liv.ac.uk

1 Institute of Integrative Biology, University of Liverpool, Biosciences Building, Crown Street, Liverpool L69 7ZB, UK

Full list of author information is available at the end of the article
}

wealth of data and manual curation efforts [11], genome annotations are largely based upon computational gene model predictions which may be inaccurate, a particular problem for complex genes containing multiple introns.

New sequencing technologies allow high-throughput sequencing of transcripts (RNA-seq) [12]. These data can be used to detect differential gene expression among different populations of cells, verify or correct gene models and identify previously unannotated transcribed regions of the genome. These include genes bound for translation as well as untranslated (non-coding) transcripts (ncRNA) that play important functional roles. Families of ncRNA include 'housekeeping' molecules such as transfer RNA (tRNA), ribosomal RNA (rRNA), spliceosomal RNA and small nuclear and small nucleolar RNA (snRNA and snoRNA). They also include ncRNA with putative regulatory roles such as small interfering 
RNA (siRNA), micro RNA (miRNA) and long ncRNA. The latter group include antisense transcripts which may be common in eukaryote transcriptomes [13], potentially playing roles in transcriptional and post-transcriptional regulation [14]. RNA-Seq has been applied to several Aspergillus species to show transcriptional responses to: biofilm growth in A. fumigatus [15]; growth on lignocellulose and germination of conidia in $A$. niger [16-18]; and temperature changes and 5-azacytidine in A. flavus $[19,20]$. These and other studies $[21,22]$ have also explored Aspergillus transcriptomes more generally and improved genome annotation.

Knowledge of the precise position of a transcript's 5' end provides valuable data for gene annotation by defining the transcription start site (TSS) and thereby both the $5^{\prime}$ untranslated region (UTR) and upstream promoter region of the respective gene. This allows the identification of functionally important sequence elements that may then be analysed directly or in silico. However, the $5^{\prime}$ end of a transcript is difficult to distinguish accurately from whole transcriptome RNA-seq data due to uneven read coverage and bias in libraries leading to a low frequency of sequence reads from transcript ends [23]. Several methods to define the $5^{\prime}$ ends of transcripts have been described, using different sequencing technologies and with varying levels of throughput [24-27].

Here, we describe both the strand specific sequencing of the whole transcriptome of $A$. nidulans and the specific sequencing of transcript $5^{\prime}$ ends. The latter was achieved by adapting 5' RATE ('robust analysis of $5^{\prime}$-transcript ends [26]). By varying the pre-treatment of RNA we were able to specifically sequence capped, uncapped or both capped and uncapped transcripts. The sequencing of transcript 5' ends led to the identification of over 7000 transcription start sites across the genome and these were used to investigate common features associated with transcription sites and promoters, including the identification of novel motifs. We found many cases of antisense transcription of protein coding genes (up to $72 \%$ of genes), as well as modest levels of alternative splicing of transcripts (up to $8.2 \%$ of genes). The occurrence of differential splicing and antisense transcripts were confirmed for specific transcripts and the regulatory role of one antisense transcript for the transcription factor meaB, investigated. These data have assisted in genome annotation, including the identification of novel genes: we estimate that the total gene number for $A$. nidulans is approximately 11,800 , over 1000 more than had previously been annotated. These data have been made available to the Aspergillus genome database (AspGD) to facilitate ongoing gene annotation and can be visualised via their genome browser.

\section{Results}

Whole transcriptome sequencing of Aspergillus nidulans

To obtain an accurate representation of the A. nidulans transcriptome, we performed high-throughput RNAsequencing of the wild-type strain G00 under a range of growth conditions [6,28]. Of 304,193,731 reads 62,213,473 (20.5\%) mapped to the reference genome, the majority mapping to only one position. $79.4 \%$ of the reference genome assembly was covered by at least one sequence read. Mapping statistics are shown in Additional file 1.

The CADRE genome assembly release 5 has 10827 annotated loci with unique identifiers. Of these, 10530 had an annotated coding sequence, indicating the translated region of a protein coding gene. The remainder consisted of putative pseudogenes and non-coding RNA genes. For all 10827 annotated loci, the number of mapped reads from each sequence library which aligned in the sense and antisense orientations were counted. These counts were normalised by library and locus size to give the standardised measure of expression 'reads per kilobase per million mapped reads' (RPKM). These data are shown in Additional file 2. Across the five libraries, $94 \%$ of annotated loci had at least one read aligned in the sense orientation and $79 \%$ had RPKM > 1 (Additional files 1 and 2), indicating that the majority (80-95\%) are expressed under the growth conditions analysed.

\section{Identification and analysis of novel transcripts}

The transcriptome alignment was used to define transcribed regions of the genome (Additional file 3). Using the merged data from all five libraries 44,479 transcribed fragments were defined. This is four times greater than the number of annotated loci. A proportion of these may relate to unanotated loci. However, multiple small fragments can be inferred from what is in fact one transcript due to uneven mapping; a particular problem if the transcript is expressed at a low level. Additionally, alternative splicing may produce multiple transcripts from the same locus.

To estimate the number of putative novel transcripts in the genome, the locations of the 44,479 transcribed regions were compared to the annotated loci. 29\% (13026/44479) did not overlap an annotated locus in either direction. On average these were shorter than transcribed fragments that overlapped annotated loci (median length $135 \mathrm{bp}$ and $580 \mathrm{bp}$, respectively; Additional file 1). The proportion of the transcribed genome associated with these novel transcripts was $8.3 \%$. Assuming annotated and unannotated genes have a similar length distribution, these data suggest that the 10,827 annotated loci represent approximately $92 \%$ of all loci. The genome should therefore contain approximately 980 additional transcripts not included in the CADRE release 5 annotation. 
To further analyse these unannotated loci, we searched for similarities to known functional non-coding RNAs or protein domains. To identify functional non-coding RNAs, the nucleotide sequences of the 13,026 putatively novel transcribed fragments were used to query the Rfam database [29]. To identify unannotated protein coding genes, we used all open reading frames longer than 300 bp (i.e. 100 aa) within these transcripts (1899 putative peptide fragments derived from 1129 different transcribed fragments) to query the Pfam database [30]. 343 transcripts had at least one match to the Pfam or Rfam database, the results are shown in Additional file 4. A few putative functional RNAs were identified, among them several tRNAs, small nucleolar RNAs and spliceosomal RNAs. Many protein domains were also identified. Since this analysis, many of these genes have been added to later releases of the annotation, in some cases guided by these data. Putative transcripts that remain unannotated at the time of writing include those encoding putative methyltransferase and P450 domains, as well as numerous DDE domains often associated with transposons.

\section{Introns and alternative splicing}

The transcriptome alignment identified 22,636 introns supported by one or more sequence reads (the locations of all identified introns from all sequence libraries are in Additional file 5). The CADRE release 5 annotation contained 24,894 annotated introns, of which 15,870 (63.8\%) were confirmed by the transcriptome data. 1087 introns matched the annotated intron only at its $5^{\prime}$ junction and 542 only at its 3' junction, while 714 overlapped an annotated intron without matching either junction. 4092 did not in any way correspond to an annotated intron.

Introns are generally short (median length $59 \mathrm{bp}$, with $95 \%$ of introns between 46 and 228 bp) but some long introns do exist. For example, an annotated intron of approximately $1.4 \mathrm{~kb}$ in gene AN4076 was confirmed by RNA-seq and validated by RT-PCR (data not shown). 21,794 introns had bases GT and AG at their 5' and 3' ends, 428 had AT and AC and 414 had GC and AG. The GT-AG introns showed standard consensus sequences at their 5' and 3' ends: GTRAAGT (base frequencies: $100,100,67,42,86,62 \%)$ with conserved AG (40, 56\%) immediately upstream at the $5^{\prime}$ end and YAG (90, 100, $100 \%)$ at the $3^{\prime}$ end. In all, 18,782 introns were fully contained within 7191 annotated genes. 227 introns extended 191 annotated genes at the $5^{\prime}$ end. 147 introns extended 140 annotated genes at the $3^{\prime}$ end. 18 introns apparently completely spanned 12 annotated loci (Additional file 1). Of these 12 loci, six were snoRNAs and two were 5.8S rRNA. The remaining four were putative protein coding genes, but the long introns could not be confirmed by RT-PCR (data not shown). Careful examination of these loci suggests that the introns are likely to represent mapping artefacts due to the respective genes being flanked by similar tRNA genes.

Alternative splicing increases the diversity of the transcriptome and proteome without the need for additional genomic sequence. A small number of examples of alternative splicing have been described in the Aspergilli [31-35]. Whole transcriptome analysis of $A$. oryzae (which was not strand specific and therefore could not account for the presence of antisense transcripts) reported $8.55 \%$ of genes displaying differential splicing with the majority of these events $(91.56 \%)$ being due to intron retention [21].

We searched for cases of alternative splicing by comparing transcripts defined by the Cufflinks program. Table 1 summarises the data from Additional file 3 . Based on these data, the proportion of alternatively spliced transcripts is low (8.3\%) and is broadly similar to that reported for $A$. oryzae [21]. We searched for cases of exon skipping/retention (two non-overlapping short introns each sharing a $5^{\prime}$ or $3^{\prime}$ boundary with a single longer intron) but found only 60 examples of this pattern, indicating that it is rare. Three of these cases were confirmed by RT-PCR (Figure 1A): AN4483, AN3433 and AN2425. We observed that many of the cases of alternative splicing involved intron retention, similar to the case in $A$. oryzae.

We observed an extreme example of alternative splicing on chromosome V. This transcript spanned three annotated loci: a putative pseudogene, a putative protein coding gene (AN11485) and a snoRNA. The putative protein coding gene was not supported by the data (no reads aligned to the same strand or supported its introns) but the snoRNA corresponded to a peak of high read coverage. The transcript was unusual in that it contained seven introns each with a peak of high read coverage within it. The alignment indicated alternative splicing with both exon skipping and retention, which were confirmed by RT-PCR. Searches of Rfam indicated

\section{Table 1 Alternatively spliced transcripts}

\begin{tabular}{llll}
\hline $\begin{array}{l}\text { Growth } \\
\text { condition }\end{array}$ & $\begin{array}{l}\text { Total } \\
\text { transcripts }\end{array}$ & $\begin{array}{l}\text { Transcript } \\
\text { sets }^{\mathbf{a}}\end{array}$ & $\begin{array}{l}\text { Alternatively spliced } \\
\text { transcript sets (\%) }\end{array}$ \\
\hline Nitrate & 35167 & 33934 & $883(2.6 \%)$ \\
Complete & 34292 & 33095 & $868(2.6 \%)$ \\
Ammonia & 36810 & 35803 & $715(2.0 \%)$ \\
$-\mathrm{N}, 4$ h & 31847 & 30552 & $932(3.1 \%)$ \\
$-\mathrm{N}, 72 \mathrm{~h}$ & 37097 & 34607 & $1743(5.0 \%)$ \\
All & 44479 & 38750 & $3226(8.3 \%)$
\end{tabular}

${ }^{\mathrm{a} A}$ set of one or more different transcripts occupying a common genomic locus and sharing regions of a common template sequence.

${ }^{\mathbf{b}}$ Transcript sets of more than one transcript (as a percentage of all transcript sets). 


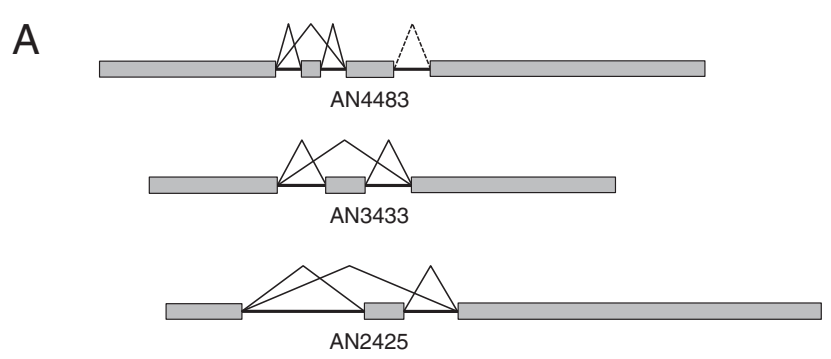

B



C

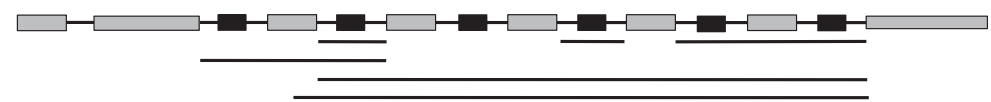

Figure 1 Alternative splicing of $\boldsymbol{A}$. nidulans transcripts. (A) Three examples of exon skipping/retention identified by whole transcriptome mapping and experimentally verified. (B) An extreme example of alternative splicing: a putative snoRNA cluster on chromosome V. Black boxes represent putative snoRNAs, grey boxes represent exons. Solid lines represent splicing events verified by RT-PCR. (C) Intron retention (solid lines beneath diagram) verified by RT-PCR and sequencing in the snoRNA cluster. The cluster displays a complex combination of splicing, intron retention and exon skipping.

that the intra-intron peaks were indeed snoRNAs and the locus appears to be a snoRNA cluster similar to that described in Neurospora crassa [36]. Figure 1B illustrates the observed alternative splicing. We could not reconstruct an open reading frame from the exon sequences, suggesting that the function of the transcript is as a precursor to fully processed snoRNAs and the processed mature transcripts may not have an additional functional role.

\section{Natural antisense transcripts (NATs)}

Inspection of the $A$. nidulans transcriptome data revealed a large proportion of genes with strand-specific reads aligned in both the sense and antisense orientations, indicating the existence of many natural antisense transcripts. These represent a subset of non-coding RNAs [37] with potential regulatory roles mediated via various mechanisms including transcriptional interference, chromatin remodelling, RNA interference and translational repression [14]. Examples have previously been identified in A. flavus and A. niger [16,38].

Loci with sequence reads mapping in the antisense orientation were relatively common, with $72 \%$ having at least 1 mapped read, compared to $94 \%$ of loci in the sense orientation (Additional file 1). However, in the antisense orientation only $14 \%$ of annotated genes had an RPKM > 1, compared to $74 \%$ in the sense orientation. This indicates that antisense transcripts are expressed at a much lower level than its sense counterpart and/or that they cover only a part of a given gene.
To describe the distribution of antisense transcription across genes, we counted antisense reads aligned to the 5', central and 3' regions of each annotated gene. Of the $72 \%$ of genes $(7697 / 10697)$ with one or more reads mapped in the antisense orientation under one or more growth conditions (Additional file 6), 352 showed 5 '-biased and 352 centrally-biased antisense transcription, but the majority, 1280, showed 3' bias. This excess of antisense transcription at the $3^{\prime}$ end of genes is consistent with previous findings in Saceromycese cerevisieae [39].

Using RT-PCR with oligo dT in combination with gene specific primers we experimentally verified antisense transcripts overlapping four genes: AN8048, AN8040, AN4023 and AN4058. PCR products were cloned and sequenced, confirming the presence of each of the previously unannotated transcripts in the antisense configuration with a known gene.

We investigated one example of antisense RNA in more detail. The transcription factor meaB (AN4900) has a regulatory role associated with nitrogen availability [40,41]. Examination of RNA-seq data revealed an antisense transcript which initiates at a position aligned to the first intron of the sense transcript. The intron sequence, which is relatively long at $331 \mathrm{bp}$ compared to a median intron length of $59 \mathrm{bp}$, contains six GATA motifs, indicating a possible functional association with a second transcription factor, AreA [42], which has been shown to regulate meaB transcript levels [41]. To confirm the presence of the antisense transcript and test the possibility that it is under the regulation of AreA, we 
utilised northern analysis using a single stranded probe from exon 1. As shown in Figure 2, the antisense transcript is differentially regulated in response to nitrogen regime and its expression is dependent on functional AreA. To investigate the function of the mea $B$ antisense transcript we specifically deleted the first intron of meaB by homolgous integration at the meaB locus. Northern analysis revealed that modulation of meaB transcript levels was largely lost, except under nitrogen starvation (Figure 2). We also found that a strain disrupted for three loci potentially involved in RNAi [43] did not show significant altered regulation of meaB transcript levels, indicating that RNAi is not responsible for antisense mediated regulation of meaB.

\section{Identification of transcription start sites and analysis of $5^{\prime}$ untranslated regions and promoters}

In order to further characterise the A. nidulans transcriptome and help improve gene annotation, we con- structed RNA-seq libraries enriched for $5^{\prime}$ TSS, utilising an approach based on $5^{\prime}$ RATE [26]. The mRNA was decapped using tobacco acid pyrophosphatase (TAP) prior to ligation of adapters to the unfragmented RNA and reverse transcription, using a primer containing a random hexonuleotide sequence at its $3^{\prime}$ end. The products were size selected, amplified by PCR and sequenced using the conventional SOLiD protocol. The resulting $5^{\prime}$ nucleotide position of each read, here referred to as the 'read head' (RH), was mapped to the genome assembly and the number of read heads at each position counted, peaks representing putative $5^{\prime}$ ends of transcripts.

The initial 5' sequencing library ('TAP-1') consisted of $64,148,556$ reads, of which 18,817,969 (29.33\%) mapped uniquely to the reference genome (Additional file 1). Inspection of the sequence alignment showed a high frequency of RH mapping close to the $5^{\prime}$ ends of annotated genes, indicating successful 5' enrichment. Additionally, widespread low level mapping was observed, possibly due

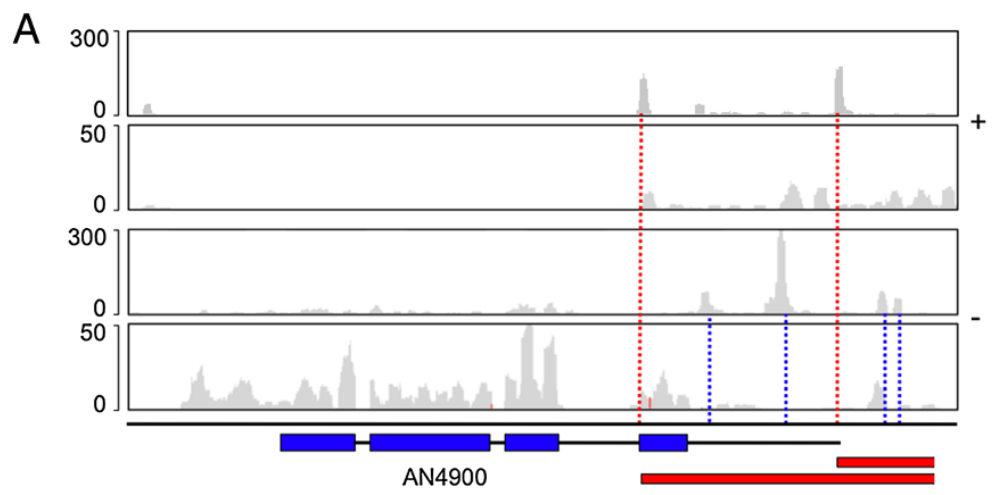

B

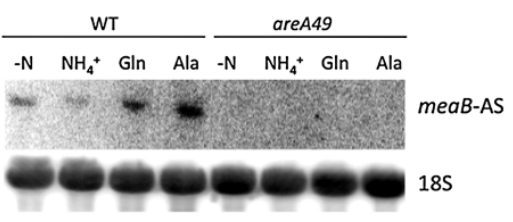

C
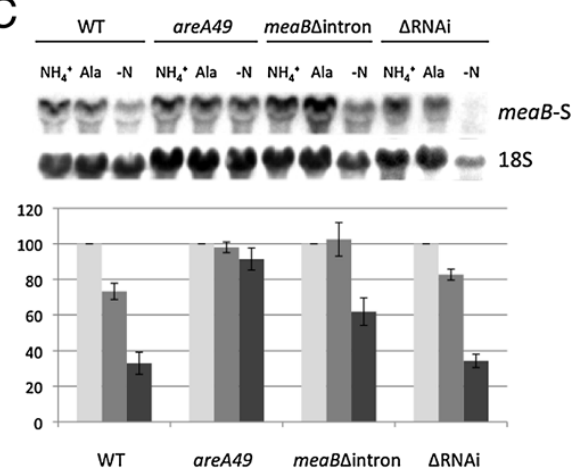

Figure 2 Antisense transcription at the meaB locus. (A) Antisense transcription was identified overlapping the $5^{\prime}$ end of the meaB gene. Coverage on the positive strand for $5^{\prime}$-specific and whole transcriptome RNA-seq libraries is shown in the top two plots. Coverage on the negative strand for these libraries is shown in the bottom two plots. The meaB gene (AN4900) is encoded on the negative strand (blue boxes and thin black lines represent exons and introns/UTR, blue dotted lines indicate multiple transcription start sites). Red boxes indicate predicted antisense transcripts, their transcription start sites shown by red dotted lines. (B) From northern analysis of the wild type (WT) the antisense transcript (meaB-AS) was identified and found to be modulated by nitrogen regime; $\mathrm{NH}_{4}^{+}$, glutamine ( $\mathrm{Gln}$ ), alanine (Ala) as sole nitrogen source or nitrogen starvation (-N), for two hours. Analysis of a strain bearing a loss of function mutation in areA (areA49) revealed that meaB-AS expression is AreA dependent. 185 rRNA was used as a control. (C) Quantitative northern analysis of the meaB sense transcript (meaB-S) in the wild type (WT), areA49, meaBAintron and $\triangle R N A i$ strains was undertaken. The mean intensity, relative to WT, of four independent experiments is plotted (+/-SD). Downregulation of meaB transcript levels in response to poor nitrogen (Ala) was lost in the meaBAintron strain but the response to nitrogen starvation was retained. 
to the sequencing of fragmented and partially degraded transcripts. To assess this we made a library in which the mRNA was pre-treated with alkaline phosphatase (AP) prior to decapping, in order to prevent fragmented or degraded RNA from ligating to the $5^{\prime}$ PCR primer. Additionally we made a library without pre-treatment of the RNA with TAP, thus eliminating capped mRNA from the sequence library. In the untreated library, low levels of reads were distributed throughout the transcribed regions with no peaks generally observed at the $5^{\prime}$ ends of annotated genes. Some peaks of high coverage were evident in the untreated library. These were often associated with the 5 ' ends of putative snoRNAs (Additional file 7). In contrast, TAP-treated libraries showed discontinuous read distributions with peaks of coverage commonly occurring upstream of annotated genes (Figure 3A). The accuracy of the transcript 5 ' end mapping was assessed by comparing it with circularisation RT-PCR or 5' RACE data, which specifically defines the $5^{\prime}$ ends of transcripts. This analysis showed agreement between the methods, as illustrated in Figure 3B.

For further analysis, to maximise the depth of coverage and identify as many putative TSS as possible, the four TAP-treated libraries (TAP-1, TAP-2, TAP-3 and AP + TAP) were combined. Using a region of 121 bp containing a minimum of $50 \mathrm{RH}$ and a minimum major peak of $10 \mathrm{RH}$, we defined 17992 putative transcription start site regions. We developed a metric to measure the distribution of reads across each TSS region, based on a confidence interval around the major TSS (described in the
Methods section). This was used to define three sets of TSS regions. The first contained 4557 putative transcription start sites with a very tight distribution or strong single start site where the $95 \%$ confidence interval was within two nucleotides. The second contained 4637 less specific single start sites (95\% confidence intervals $>2$ and <4). The third contained the 8798 most diffuse TSS (95\% confidence intervals $>4$ ). Of the tight, intermediate and diffuse TSS regions, $72.3 \%$ (3293), 46.8\% (2171) and $25.0 \%$ (2197) were within 500 bp upstream of annotated genes. Conversely, 11.9\% (542), 23.4\% (1087) and 40.9\% $\%$ (3595) occurred fully within annotated genes, suggested that the tight TSS regions are likely to be enriched for real TSS. However, the diffuse TSS regions might include a proportion of artefacts arising from degradation of mRNA. All putative promoters are recorded in Additional file 8 and all identified TSS located within $500 \mathrm{bp}$ of the $5^{\prime}$ end of an annotated gene and in the correct orientation are listed in Additional file 9. According to our classification of TSS, 3293 'tight', 2172 'intermediate' and 2197 'diffuse' putative TSS were associated with annotated genes.

The association of specific gene functions with different classes of promoter was investigated. Figure 4 shows the proportion of each of the three gene sets assigned a broad functional description. Genes associated with 'tight' TSS are enriched for functions related to translation and ribosome biogenesis, with genes associated with diffuse TSS tend to be enriched for

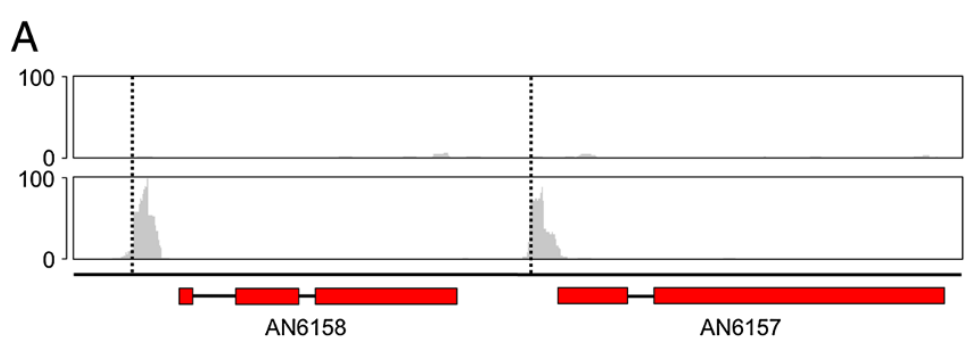

B

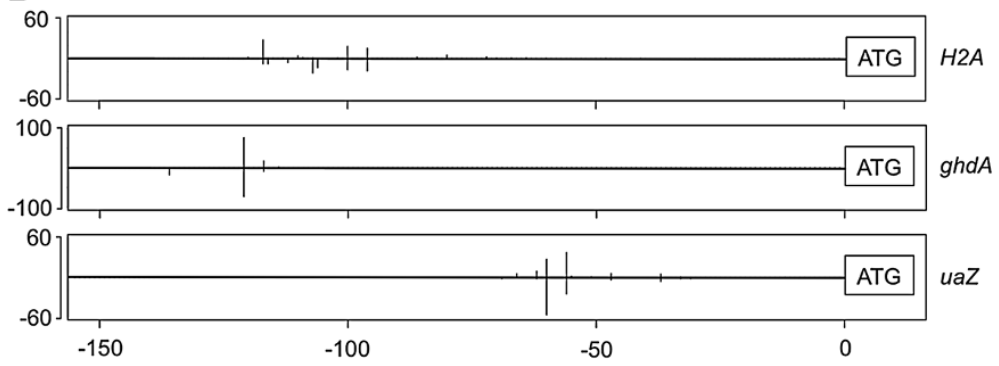

Figure 3 5'-specific RNA-seq locates transcription start sites. (A) Coverage depth of 5'-specific RNA-seq libraries (plotted in grey). Peaks and dotted lines indicate the transcription start sites of two annotated genes: AN6158 and AN6157. (B) Experimental validation of 5'-specific RNA-seq data. The $x$-axis indicates the distance (in base pairs) upstream of the translation initiation codon ATG for three genes: H2A.Z (AN8039), gdhA (AN4376) and uaZ (AN9470). For each gene, the proportion of RNA-seq reads starting at a given position is plotted above the line and the approximate proportion of 30-50 Sanger-sequenced plasmids containing products of circularisation RT-PCR supporting a given TSS is potted below the line. 


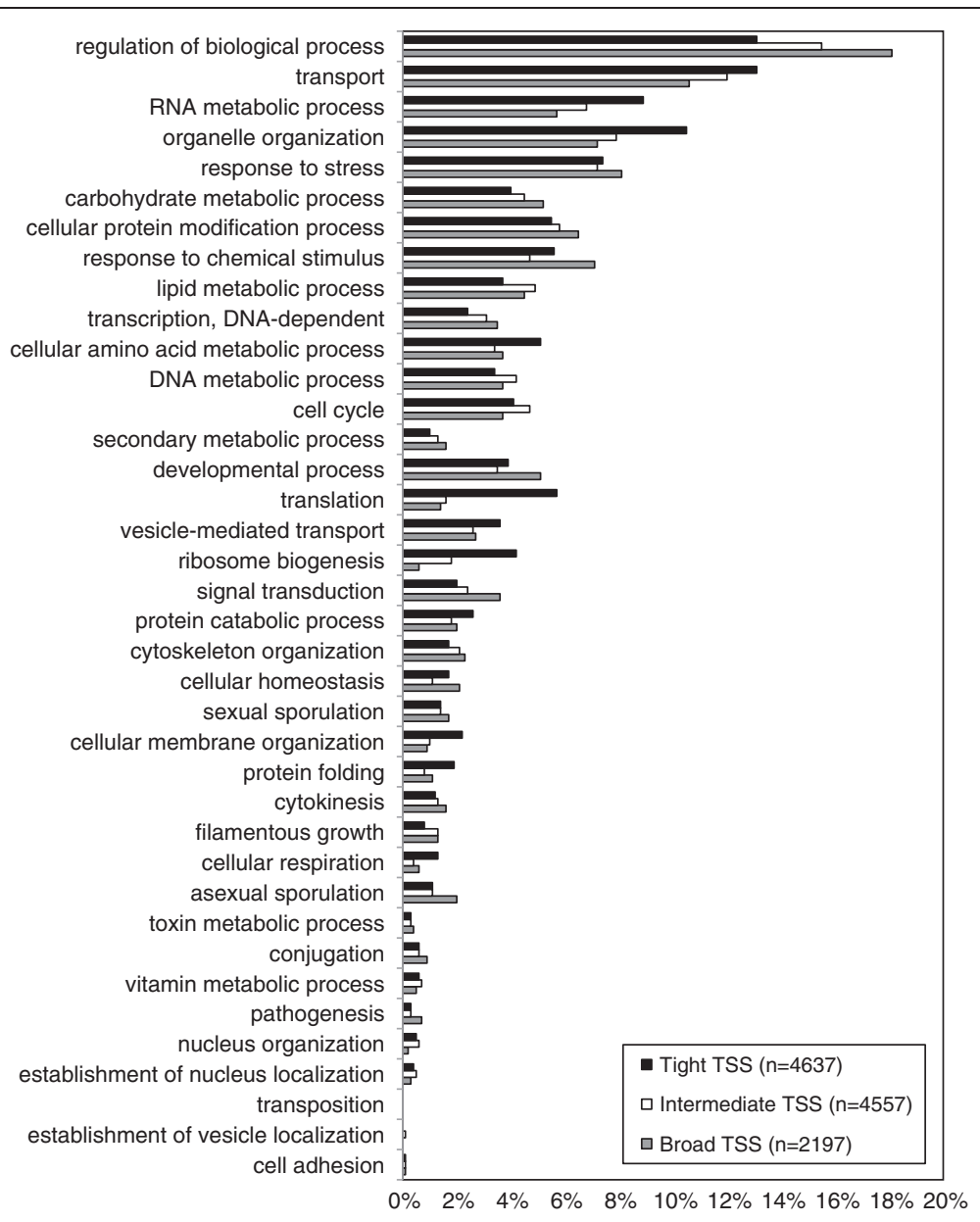

Figure 4 Functional description of genes associated with different TSS classes. The proportions of gene sets associated with different classes of TSS ('tight', 'intermediate' and 'diffuse') with general functional gene ontology descriptions. In total, 4637, 4557 and 2197 genes were asociated with 'tight', 'intermediate' and 'diffuse' TSS, respectively. Of these genes, the proportion of each set associated with a given GO term is plotted (note that genes can be associated with more than one GO term). Differences among the frequencies of GO terms reflect their overall frequency distribution across the genome. In addition, the frequencies of some functional descriptions vary among the different TSS classes. For instance, translation and ribosome biogenesis are distinctly skewed towards genes with tight TSS.

regulatory processes and signal transduction. The full table of GO terms significantly enriched for each gene set is in Additional file 10.

To define the length of $5^{\prime}$ UTRs, the distance from each defined transcription start site to the nearest downstream annotated translation start codon (up to a maximum of 500 bp away) was plotted (Figure 5). The majority of 'tight' TSS were followed by a 5' UTR of less than $100 \mathrm{bp}$ (median $93 \mathrm{bp}$, with 95\% of UTR between 15 and $434 \mathrm{bp})$. However, a proportion of genes with long 5' UTR would not be detected here, as we set a maximum distance of $500 \mathrm{bp}$ from TSS to translation start codon to minimise false positive results. For the more diffuse TSS, the distribution 5' UTR length was much broader (data not shown). To characterise upstream promoter regions, we analysed regions $200 \mathrm{bp}$ upstream and 25 bp downstream of the putative TSS. Figure 6 shows the

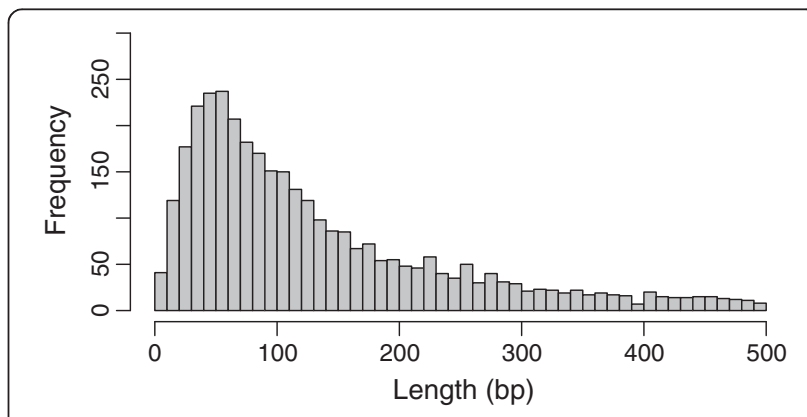

Figure $5 \mathbf{5}^{\prime}$ UTR lengths. Length distribution of $5^{\prime}$ untranslated regions (between the transcription start site defined by $5^{\prime}$-specific RNA-seq and the annotated translation initiation codon) for 'tight' transcription start sites. 


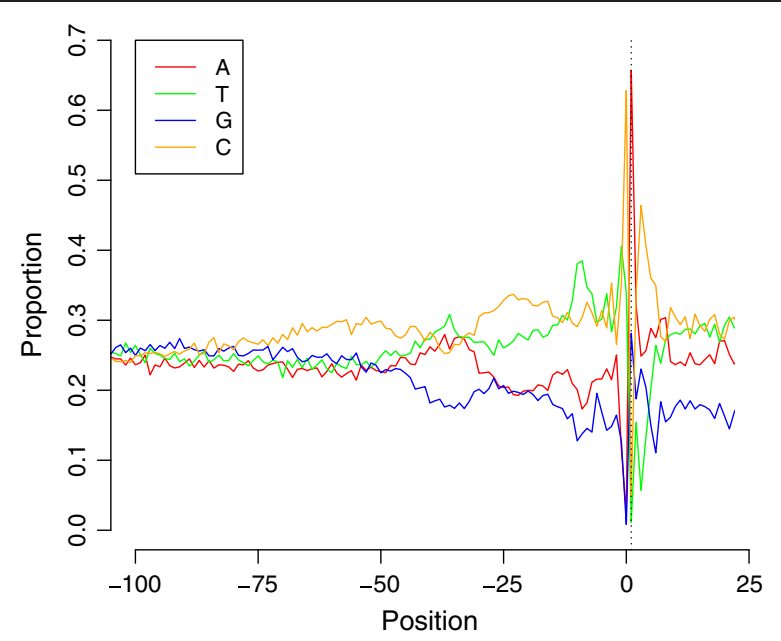

Figure 6 Base composition around transcription start sites. The proportions of bases from 100 bp upstream to 25 bp downstream of putative 4637 'tight' transcription start sites (100-200 bp upstream shows no perturbation from the 25\% expected by chance and is not shown). The region is enriched for $C$ from approximately position -75 and for $C$ and $T$ from approximately position -50 . A peak of $A$ and $T$ is seen in the region -45 to -30 , which may indicate putative TATA box motifs. Nucleotide $C$ peaks at position -1 and nucleotide $A$ at the transcription start site, followed by $\mathrm{C}$.

base composition and Figure 7 the dinucleotide composition of these regions for the 4637 'tight' TSS (data not shown for the less well defined 'intermediate' and 'diffuse' TSS sets, but are broadly similar to data for the 'tight' TSS set). The plots indicates that base and dinucleotide composition is skewed within approximately 75 bp immediately upstream of the TSS, indicating that functional elements of the core promoter may lie within this region. Further upstream (position -100 to -200 ) showed no major skewed base/dinucleotide frequencies (data not shown). The major features that can be discerned include a C-rich region extending approximately from position -75 to -50 and a generally pyrimidine (TC)-rich region spanning the TSS from -50 to at least position +25 . Immediately upstream of the TSS, $\mathrm{C}$ is the most common nucleotide (62.9\% of tight promoters), followed by $\mathrm{T}, \mathrm{A}$ and $\mathrm{G}(33.9 \%, 2.4 \%$ and $0.8 \%)$. At the TSS itself, $\mathrm{A}$ is the most common base $(65.7 \%$ of tight promoters), followed by G, C and $\mathrm{T}(28.1 \%, 5.0 \%$ and $1.2 \%)$. Reflecting this, CA is the most frequent dinucleotide spanning the TSS $(49.1 \%$ of tight promoters), followed by TA, TG and CG (16.0\%, $14.4 \%$ and $12.7 \%$ ). All other dinucleotides here are each at less than $5 \%$ frequency. Other peaks include TT/TC approximately $-10 \mathrm{bp}$ upstream of the TSS and an A and $\mathrm{T}$ rich peak in the region approximately -45 to $-30 \mathrm{bp}$ upstream of the TSS, which may represent the site of the TATA box.

To identify functional motifs in the upstream promoter regions, we searched for statistically over-represented strings of bases within the promoters of tight, intermediate and diffuse TSS using the program YMF [44]. Similar results were obtained from the program MEME (data not shown) [45,46]. The full results from YMF are shown in Additional file 11. From this analysis, we identified a large number of variants on three common sequences: CACGTG, ACCGCC and CCTNAGG. We investigated the distribution of these motifs relative to the 4637 'tight' TSS (Figure 8) and each was clearly not uniform (chi-squared test of uniformity: $\mathrm{P}<2.2 \times 10^{-16}$ in all cases) occurring primarily more than $50 \mathrm{bp}$ upstream of the TSS. The marked non-random distribution of these putative motifs relative to the TSS suggests that their location is relevant to their function. Our analysis of enriched motifs did not identify known core promoter motifs such as the TATA box (TATAA) so we searched for this motif individually in the promoter regions (Figure 8). The distribution of putative TATA box motifs was strongly positionally specific, with the majority occurring 30-45 bp upstream of the TSS, consistent with the skewed A and T frequencies in this region. We also searched for motifs including the CAT box (CCAAT), initiator element (YYANWYY), heat shock elements (NGAANNTTCN) [47] and regulatory motifs bound by CreA (SYGGGG) [48] and AreA (HGATAR) [49]. Their positions were generally much less well defined than for the other motifs although the InR and CAT box tended to be more common near to the TSS while the CreA and AreA motifs tended to be more common between -50 and -100 (Additional file 1).

To further validate the putative motifs CACGTG, CCTNAGG and ACCGCC, we investigated whether they were conserved among the distantly related species Aspergillus nidulans and Aspergillus fumigatus. We defined 5872 orthologue pairs between the two species and utilising chi-squared analysis determined if co- 

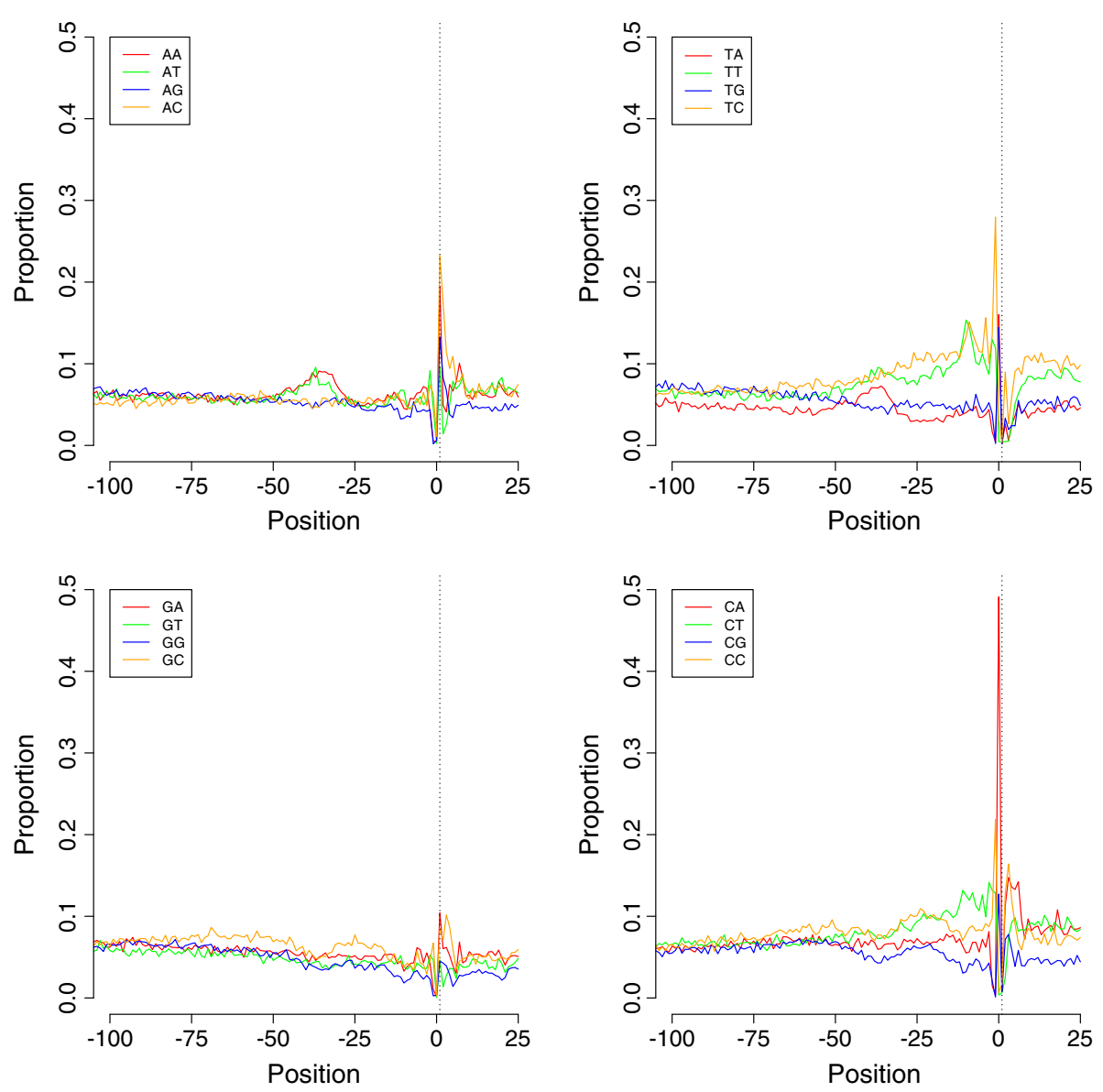

Figure 7 Dinucleotide composition around transcription start sites. The proportions of all possible dinucleotides from $100 \mathrm{bp}$ upstream to 25 bp downstream of 4637 putative 'tight' transcription start sites (100-200 bp upstream shows no perturbation from the 6.5\% expected by chance and is not shown). A small peak in AA, AT and TA frequencies in the region -45 to -30 may represent the TATA box. From -50 to +25 around the transcription start site is enriched for pyrimidine pairs (TT, TC, CT, CC) and the TSS and position - 1 is strongly enriched for CA, reflecting the single nucleotide frequencies at these positions.

occurrence in orthologous promoters was significant. For all three motifs this was the case (CACGTG, $\mathrm{p}<2.2 \times$ $10^{-16}$; ACCGCC, $\mathrm{p}<2.2 \times 10^{-16}$; CCTNAGG, $\mathrm{p}=3.17 \times$ $\left.10^{-10}\right)$. This is consistent with all three motifs being functionally conserved. Sequence alignment of upstream regions for orthologous genes from multiple species also illustrates the phylogenetic conservation of motifs (Figure 9 and Additional file 1).

\section{Discussion}

We carried out sequencing of both the whole transcriptome and transcript $5^{\prime}$ ends from the filamentous fungus $A$. nidulans using five different growth conditions. Combining these two methods provides a useful way to define transcript structure and to improve genome annotation.

We observed that at least $80 \%$ of the reference genome assembly was transcribed across all five conditions tested. While these proportions should be treated with some caution as they are sensitive to factors such incomplete sampling by smaller sequence libraries, such effects will lead to underestimation so we may still conclude that the majority of the genome is transcribed. Furthermore, this will inevitably be extended when further growth regimes are examined. The developmental state of the cultures was not measured but some growth conditions, particularly nitrogen starvation, can induce sporulation in submerged cultures [50]. Our data suggest that this is the case, as $\operatorname{brl} A$ and several other genes up-regulated during condia formation [51] appear to be upregulated in cells under long term nitrogen starvation (Additional file 2). This may in part explain why more of the genome (approximately 71\%) appears to be transcribed under nitrogen starvation compared to other conditions (54-61\%). However, for all conditions, more than $50 \%$ of the genome was transcribed. The annotated protein coding portion of the genome is approximately $50 \%$, indicating a large amount of functional non-coding 

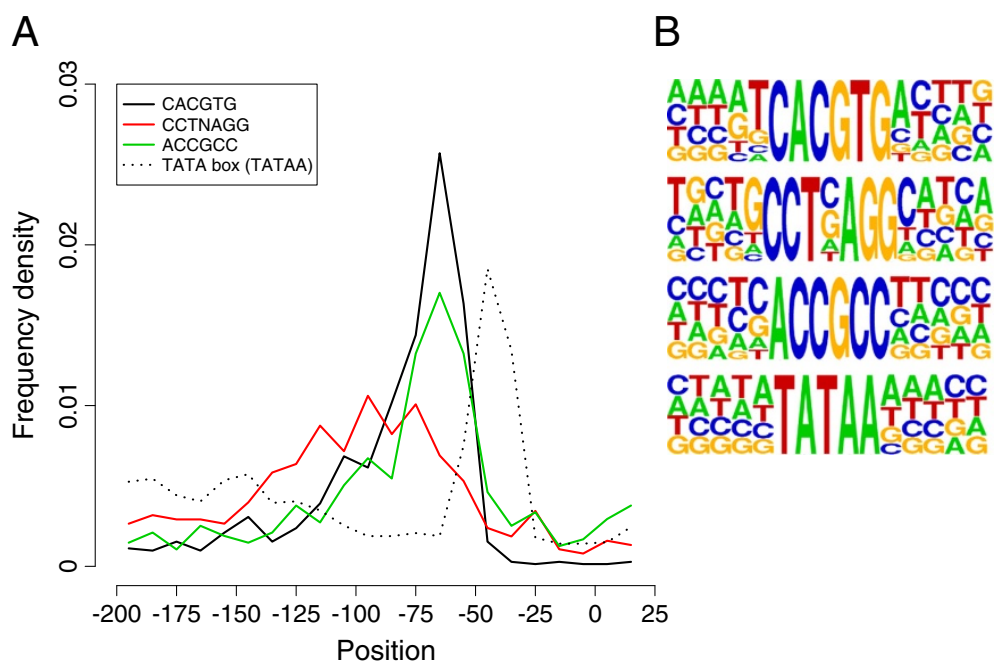

Figure 8 Distribution of putative promoter motifs relative to transcription start sites. (A) Distributions of consensus motifs CACGTG, CCTNAGG and ACCGCC within promoter regions of 'tight' transcription start sites. The distribution of putative TATA box motifs (TATAA) are also shown. Each point represents the frequency density of motifs starting within a $10 \mathrm{bp}$ window, the midpoint of which is plotted. Both CACGTG and ACCGCC peak at approximately -70 bp and the TATA box at $-45 \mathrm{bp}$ from the TSS, while the position of the CCTNAGG motif is less sharply defined across a region from approximately $-70 \mathrm{bp}$ to $-120 \mathrm{bp}$. (B) Sequence logos of promoter motifs and surrounding nucleotides (from promoter regions associated with 'tight' TSS).

RNA in addition to this (including functional noncoding transcripts in addition to untranslated regions of protein-coding genes). In this, $A$. nidulans resembles $S$. cerevisiae, in which approximately $75 \%$ of the genome encodes proteins but $85 \%$ of the genome is transcribed [39]. More extreme pervasive transcription is displayed by mammalian genomes (e.g. [52-55]), in which the difference between protein coding sequence (generally $<5 \%$ of the genome) and total transcribed sequence (70-90\%) far exceeds that seen in the more gene-dense fungi.

Updating and improvement of the A. nidulans genome annotation is an ongoing process and combining whole transcriptome sequencing with $5^{\prime}$ specific RNA-seq data can help to improve gene annotation. As an example, a gene which had been mis-annotated as two separate loci was identified due to the occurrence of only a single TSS and identification of previously unannotated introns (Figure 10). To aid the annotation process, the RNA-seq data were made available to the research community via the CADRE and AspGD websites [7,8]. At AspGD, read alignments can be viewed (using the JBrowse feature on each gene page) and the data have been used to update many of the gene model annotations on AspGD (where this is the case is indicated in the 'locus history' section of the gene page). Consequently, the current AspGD annotation has changed considerably since that analysed






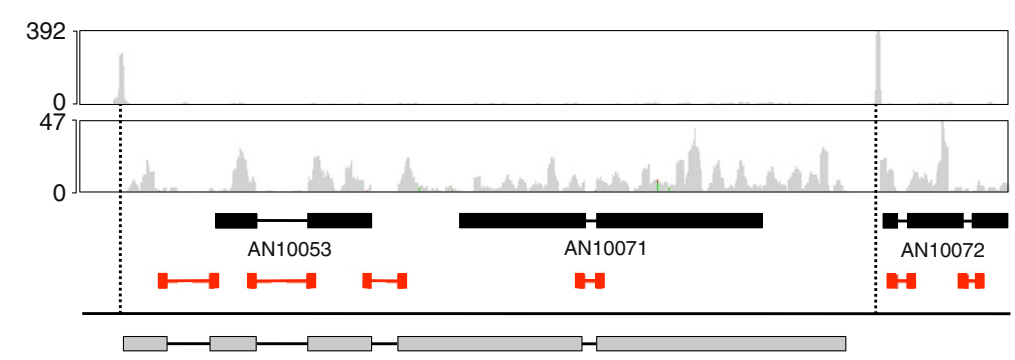

Figure 10 Gene annotation improvement guided by RNA-seq. Example of a correction of misannotated gene models. In the CADRE release 5 annotation two genes, AN10053 and AN10071, were predicted (thick and thin black bars represent exons and introns). Whole transcriptome (middle track, coverage depth plotted in grey) and 5'-specific RNA-seq (top track, coverage depth plotted in grey) revealed the general structure of transcripts produced in this region. Whole transcriptome coverage identified four introns in the region covering the two genes (red), two previously unidentified introns extended AN10053. 5'-specific sequencing identified a single 5' end for both annotated genes (left dotted line). The combined data indicates a single gene (bottom, grey boxes and black lines represent exons and introns).

here. For instance, we found many putative unannotated protein genes in our analysis and estimated that the total number of transcribed genes is close to 11,800 (approximately 1000 more genes than in the annotation we analysed). Many of these putative genes have since been annotated.

Transcriptome complexity was extended by many examples of alternative splicing. Predominantly this involved intron retention which we found generally resulted to the inclusion of an in-frame stop codon into the transcript. Therefore, we hypothesise that intron retention is not primarily a means of generating novel proteins, but is linked to repression of translation. This could be achieved by rapid nonsense mediated decay of transcripts containing premature stop codons [56], or intron retention could prevent export of the transcript from the nucleus [57].

We observed many instances of natural antisense transcripts (NATs). NATs are potentially functional long non-coding RNAs that may regulate protein expression via a number of possible mechanisms: transcriptional interference, chromatin remodelling, and doublestranded RNA formation leading to translational repression, RNAi or disrupting mRNA maturation and processing. A number of transcriptome analyses have identified NATs in fungi $[16,18,58,59]$. Up to $90 \%$ of $S$. cerevisiae genes show some level of antisense transcription and the pattern of antisense transcription appears to be evolutionarily conserved among multiple yeast species, suggesting an important conserved gene regulatory mechanism $[58,59]$. In $A$. nidulans we found that $72 \%$ of genes have at least some antisense transcription, with $14 \%$ showing this to be relatively abundant $(>1$ RPKM). Despite the importance of antisense transcripts suggested by this ubiquity and conservation, validated examples of antisense mediated regulation are limited, although artificial antisense constructs have been used to knock down gene expression in various species including $A$. nidulans [60]. In this respect we have presented data for the meaB loci, which supports the model whereby the antisense transcript is upregulated when the sense transcript is repressed and that elimination of the antisense transcript leads to loss of this transcriptional regulation. With regards to the regulatory mechanism involved in this case we found that disruption of the genes associated with RNAi [43] did not have an impact on meaB transcript levels. From our data in relation to mea $B$ there is no evidence for splicing being affected. It is interesting to note that the orthologous gene in Fusarium fujikuroi, has a similar, relatively large intron containing GATA motifs and a functional promoter [41]. In this case the resulting short transcript, which is not likely to code a functional protein, is also upregulated under nitrogen limitation but is transcribed in the sense and not the antisense direction. The apparent functional conservation would possibly support chromatin structure playing a key role in this regulatory mechanism.

The prevalence of antisense transcripts has important consequences when monitoring gene transcription. Conventional northern or quantitative PCR (qPCR) approaches do not take this into account, although in the case of northerns it is likely that the different forms of transcript identified by a probe will be distinguished on the basis of size. However, qPCR will generally not distinguish between sense and antisense unless the primers are specifically designed to avoid antisense transcripts on the basis of location or splicing patterns. As the sense transcript and antisense can be differentially regulated, very significant errors in calculating transcript levels may result as a consequence.

5'-specific RNA-seq allowed us to identify a large number of putative transcription start sites. A range of patterns of TSS distribution was seen, from tightly defined TSS with a single, well supported position to more diffuse collections of individually less well 
supported TSS. The occurrence of multiple distinct TSS, and consequent $5^{\prime}$ heterogeneity, has been described in many eukaryotic organisms, including fungi [61-65]. We divided putative TSS into three groups: 'tight', 'intermediate' and 'diffuse', based on the distribution of reads. RNA polymerase II-dependant promoters of vertebrates have previously been divided into two groups, categorised as either TATA or CpG types, the former with a tight TSS a strict distance from the TATA box and the latter with broad clusters of TSS [62,66]. The Drosophila melanogaster genome has both tight and broad TSS clusters, tight promoters appear to be enriched for core promoter elements such as the TATA-box, however Drosophila lacks CpG islands, therefore its broad promoters are not CpG-associated [67]. In Arabidopsis thaliana the TATA promoter is also associated with sharp TSS clusters [68]. In our analysis of $A$. nidulans, we found putative TATA-box motifs (TATAA) upstream of $21 \%$ of 'tight' TSS, $17 \%$ of 'intermediate' TSS and $11 \%$ of 'diffuse' TSS, approximately 30 to 45 nucleotides upstream of the start site, suggesting some enrichment in tight promoters.

To analyse the core promoter regions, we focused our study on 4637 well defined 'tight' TSS. The core promoter serves as the site of organization of the basal transcription machinery, including RNA polymerase II. Based on the defined transcription start sites, we analysed upstream regions to characterise these putative promoters. Based on the skewed distribution of nucleotides, we suggest that the promoter regions are quite restricted, commonly extending no further than 75 bp upstream of the transcription start site. This is consistent with the relatively small intergenic regions of $A$. nidulans, compared to, for instance, mammalian genomes. These core promoters are generally enriched for pyrimidines (particularly between position -50 and -1 and also in the $5^{\prime}$ UTR to at least 25 bp downstream of the TSS) and an $\mathrm{A}$ and $\mathrm{T}$ rich region between -45 and -30 may represent the TATA box. The pyrimdine rich sense strand upstream of the TSS has been noted previously [69]. Most strikingly the TSS are commonly defined by the dinucleotide CA (49\% of 'tight' TSS), with A as the first base of the transcript. There are no universal core promoter motifs identified in eukaryotes, but some, such as the TATA box, are present in $30-40 \%$ of promoters in higher eukaryotes. The TATA box and initiator (Inr) element tend to have a relatively fixed location upstream of genes [70]. Although neither of these elements were identified by de novo analysis of promoter sequences based on motif enrichment they did show distinct distributions which are indicative of their functional role within promoters and also supports validity of our data.

We identified a small number of putative core motifs that were over-represented in promoters and showed a strong, significant bias in their location upstream of the TSS. They also appeared to show greater than expected conservation among divergent Aspergillus species (e.g. A. fumigatus and A. oryzae proteins share approximately $66-67 \%$ sequence similarity with $A$. nidulans [6]). Taken together, this is consistent with them being promoter elements. Further experimental analysis will be required to assess their functions - key to this will be whether they are involved in modulation of promoter activity or are, like the TATA box, primarily associated with the core function, defining the promoter start site.

\section{Conclusions}

Transcriptome sequencing, including 5' specific analysis, has revealed the complexity of the $A$. nidulans transcriptome. We found alternatively spliced genes, intron retention and many cases of overlapping transcripts. In one example, meaB, we have shown the antisense RNA has a regulatory role. We also identified many previously unannotated genes. Global analysis of $5^{\prime}$ transcript ends provides an extensive map of promoters across the genome, defining key features and facilitating future analysis of promoter structure and function.

\section{Methods}

\section{Growth of Aspergillus nidulans and RNA extraction}

All analyses used the Aspergillus nidulans wild-type strain designated G00 in the Glasgow collection. This is the strain used for the genome sequencing project (in that case named FGSC A4) [6]. Mycelia were grown in an orbital incubator at $190 \mathrm{rpm}$ for 16 hours at $37^{\circ} \mathrm{C}$ in batch cultures of $250 \mathrm{ml}$ under five different growth conditions: complex media or synthetic minimal media with nitrate as the sole nitrogen source; ammonia as the sole nitrogen source; nitrogen starvation for four hours; nitrogen starvation for 72 hours. Both minimal synthetic or complete media [71] were used with glucose $(1 \% \mathrm{w} / \mathrm{v})$ as sole carbon source. For minimal media nitrogen was supplied as either $\mathrm{NaNO}_{3}$ (nitrate) or ammonium tartrate $(10 \mathrm{mM})$. For nitrogen starved cultures initial growth was conducted over night with nitrate. The mycelia were then harvested by filtration through Miracloth (Calbiochem) and washed with fresh, warm, nitrogen free media prior to re-inoculation and further incubation for either 4 or 72 hours.

Harvested mycelia were washed with cold water, excess liquid was removed on blotting paper and mycelia were snap-frozen in liquid nitrogen. Total RNA was extracted by cryogenic grinding and successive rounds of purification by phenol:chloroform and lithium chloride precipitation as described previously [72]. Poly(A) + RNA was isolated by oligo(dT) selection using the Oligotex direct mRNA kit (QIAgen), following the manufacturer's protocol. 


\section{SOLiD RNA-seq library preparation}

For total mRNA-sequencing libraries, poly(A) + selected RNA was decapped with Tobacco Acid Pyrophosphatase (Epicentre) and fragmented with RNaseIII (ABI). Strand specific cDNA libraries were constructed using oligo-dT selected mRNA and sequencing libraries prepared according to the standard protocol for SOLiD Total RNA-seq and barcoding kits (ABI).

5' specific RNA-seq libraries were prepared from samples of oligo-dT selected RNA. RNAs were decapped with Tobacco Acid Pyrophosphatase (Epicentre). The RNA was not fragmented prior to SOLiD adaptor ligation which was performed as for Total RNA-seq libraries. By excluding the fragmentation step, full length transcripts containing the SOLiD P1 PCR primer at the $5^{\prime}$ end. cDNA were produced by RT PCR with Superscript III (Invitrogen) and a random primer consisting of the internal adaptor sequence of the SOLiD P2 adaptor with a $5^{\prime}$ hexomeric degenerate sequence $\left(5^{\prime}\right.$-CTGCC CCGGGTTCCTCATTCTCTNNNNNC-3'). The resulting cDNA libraries consisted entirely of random length fragments representing the $5^{\prime}$ end of an mRNA molecule with the full SOLiD P1 adaptor and the internal adaptor sequence of the SOLiD P2 adaptor. cDNA libraries were purified, size-selected and amplified as for Total RNA-seq library preparation and subjected to the maximum recommended number of 18 PCR cycles using reagents from the ABI Total RNA-seq and barcoding kits (ABI).

Additional libraries were prepared to sequence only the $5^{\prime}$ ends of full-length, capped RNAs. The protocol used was as above, with the addition of rAPid alkaline phosphatase (Roche) treatment prior to decapping. Alkaline phosphatase removes the phosphate group from the $5^{\prime}$ ends of RNAs where no cap structure is present, thus excluding natively decapped or fragmented RNAs from the final sequencing library. Reactions were stopped by phenol: chloroform extraction.

\section{SOLiD sequencing and data mapping}

All libraries were aligned to the Aspergillus nidulans FGSC A4 genome sequence [6] release 5 (20-05-2010) downloaded from the Ensembl Genomes ftp site [9]. The 5' TSS-enriched RNA-seq libraries were sequenced on the ABI SOLiD version 3 plus. 5' TSS sequence reads were aligned to the reference sequence using Bowtie version 0.12.7 [73]. Whole transcriptome RNA-seq libraries were sequenced on the ABI SOLiD version 4. Whole transcriptome sequence reads were aligned to the reference sequence using Tophat version 1.3.1 [74] to allow mapping of reads across splice junctions. Tophat was run initially without any guidance from reference annotation, using default parameters except for a maximum intron length (option ' $-\mathrm{I}$ ') of 4000 and minimum intron length (option ' $-\mathrm{i}$ ') of 10, to disallow the prediction of unrealistically long introns. Predicted introns from the unguided mapping were combined with predicted introns from the annotation and Tophat was run again with these intron locations as a guide. This method ensures that these putative splice junctions are specifically tested and improves intron finding performance.

All sequence read and quality data were submitted to the European Nucleotide Archive under the project accession number PRJEB4484. In addition, the data were made publicly available online through the Central Aspergillus Data REpository (CADRE) website [8,75] and the AspGD website $[7,76]$.

\section{Bioinformatic analysis of novel transcripts and antisense transcription}

Transcript prediction based on the Tophat alignment of whole transcriptome data was performed using Cufflinks version 1.3.0 [77]. Alignment files were split to separate reads mapped to the forward and reverse strands and Cufflinks was run, using default parameters, on each file separately. Comparison of predicted transcripts to the A. nidulans CADRE release 5 genome annotation (to identify unannotated transcripts) was done using custom perl scripts.

To assess the protein coding potential of unannotated transcripts, open reading frames were identified and translated using the 'getorf' program, part of the EMBOSS suite of sequence analysis software [78]. A minimum ORF length of 300 nucleotides in either direction was specified. Translated ORFs were used to search the Pfam database of protein domain hidden Markov models via the web server $[30,79]$.

To analyse antisense transcription, we measured the distribution of antisense reads aligned to annotated loci. We split each locus into $5^{\prime}$, central and $3^{\prime}$ thirds and defined loci as $5^{\prime}$-biased $(>40 \%$ of antisense reads mapped $5^{\prime},<10 \%$ mapped $3^{\prime}$ ), centrally biased ( $>75 \%$ of antisense reads mapped centrally) or $3^{\prime}$-biased ( $>40 \%$ of antisense reads mapped $3^{\prime},<10 \%$ mapped $\left.5^{\prime}\right)$.

\section{Confirmation of alternative splicing}

Example of genes for which the RNA-seq alignment indicated multiple transcript isoforms due to alternative splicing were validated by RT-PCR. We designed primers to detect transcript fragments of different length across the putative alteratively spliced region of three genes: AN4483 (forward 5'-CAAGGATGCTTCCGGTGA-3' and reverse 5' -CAGAGCTCGAGGACAATG-3'); AN3433 (forward 5'-CCAATCAACACCGTCCTC-3' and reverse 5'-TGCAGGCCATTGACTAGC-3'); AN2425 (forward 5' TCTGGGGTCTGATGTTCC-3' and reverse 5'-CAGCG GCTGACGACAAAA-3'). Total RNA was extracted from wild type G00 A. nidulans grown on both minimal media 
in the presence of sodium nitrate as a sole nitrogen source and on nutrient limited media (oat) as an extreme alternative condition where metabolic stress should promote alternative splicing of transcripts. RNA samples were treated with DNaseI followed by phenol/chloroform purification to remove genomic DNA contamination. Reverse transcription was performed using an oligo-dT primer (25mer) and Superscript III reverse transcriptase (Invitrogen), according to the manufacturer's protocol, followed by PCR. PCR products were run on a $2 \%$ agarose gel stained with ethidium bromide and visualised under UV and their sizes determined using a $50 \mathrm{bp}$ ladder.

\section{Confirmation of novel natural antisense transcripts}

RNA extraction and RT-PCR were carried out as described above. Specific primers were designed for transcripts antisense to four annotated genes: AN8048 (5' - GTCCGCCAGATATACTA-3'); AN8040 (5'-GAAC TGGCTCTTAATG-3'); AN4023 (5'-ATCACCGAACT GAGACT-3'); AN4058 (5' -CCACCGTATATCATCAG$\left.3^{\prime}\right)$. These were used in combination with an oligo-dT (25mer) primer to amplify approximately $200 \mathrm{bp}$ at the 3 ' end of each transcripts. 30 rounds of PCR were performed with and KOD hot-start polymerase (Novagen) according to the manufacturer's protocols. PCR products were separated on a $1.5 \%$ agarose gel and bands around 200 bp length excised and purified using the QIAquick gel extraction kit (QIAgen). DNA fragments were ligated into pGEM-T Easy vectors (Promega) and transformed into competent $E$. coli which were grown on selective media containing ampicillin. Plasmids were extracted using a plasmid extraction kit (QIAgen). Sanger sequencing was used to confirm the identity of the amplified fragments.

\section{Defining $5^{\prime}$ transcription start sites}

Mapped 5' TSS read coverage data were converted to read head $(\mathrm{RH})$ frequencies. Read heads are the base 1 position up or downstream of the $5^{\prime}$ start position of each read mapped to the genome (the 1 bp offset accounts for the single base trimmed off the $5^{\prime}$ end of the read during mapping). Visual inspection of the alignment indicated that in the majority of cases, a major peak was surrounded by lower peaks within approximately $60 \mathrm{bp}$ on either side. We therefore defined a transcription start site region as a region of $121 \mathrm{bp}$ centred on a major $\mathrm{RH}$ peak (minimum depth $10 \mathrm{RH}$ ) with a minimum of $50 \mathrm{RH}$ mapped within it. This captured 'tight' TSS regions with a single peak, as well as more 'diffuse' TSS regions with multiple, lower peaks.

We sought a way to quantify how tight or diffuse the 'distribution of each transcription start site: whether there was a single, well defined, start site or a series of alternative start sites spread over a given area. If $\mathrm{RH}$ frequency peaks were normally distributed around the TSS then the mean would be the main TSS and a confidence interval would define the distribution around it. However, RH frequencies within 121 bp TSS regions were not normally distributed but often a number of randomly dispersed and sized peaks occurred within this window. We found that we could calculate an analogous confidence interval metric if the data were adapted by reversing the region around the peak position and adding the $\mathrm{RH}$ frequencies of the reversed region to those of the original to create an approximately normal distribution while maintaining the variance of the unedited data. The relationship between variables could then be expressed by the following equation:

$$
\mathrm{CIL}=\mathrm{z} 2 \sigma / \sqrt{\mathrm{n}}
$$

where $\mathrm{z}$ is the upper percentage point of the standard normal distribution and confidence interval length (CIL) is directly proportional to the standard deviation of $\mathrm{RH}$ distribution $(\sigma)$ and inversely proportional to the sample size (n). The distributions of adapted TSS regions are still non-normal and many of sample sizes were small, so a bootstrap method, which does not assume any underlying distribution, was applied to evaluate CIL. We used this metric to group TSS regions into 'tight' $(\mathrm{CIL}<=2)$, 'intermediate' $(2<\mathrm{CIL}<=4)$ and 'diffuse' (CIL > 4).

\section{Identification of putative transcriptional regulatory motifs} In order to identify putative transcriptional regulatory motifs in upstream promoter regions, we used the TSS data to define promoters. For 'tight' $(\mathrm{CIL}<2)$, 'intermediate' $(2<\mathrm{CIL}<4)$ and 'diffuse' (CIL > 4) TSS, a region $200 \mathrm{bp}$ upstream and $24 \mathrm{bp}$ downstream of the main TSS (the central, highest $\mathrm{RH}$ frequency peak) was analysed. For the diffuse promoters, to further reduce artefacts, only promoters within $500 \mathrm{bp}$ upstream of an annotated gene were analysed.

Putative functional motifs in these promoters were identified using YMF v3.0 [44]. YMF constructs a thirdorder Markov model to identify motifs enriched in a set of sequences. Enrichment is assessed relative to a 'background' based on similar genomic sequence. This background was a set of 10529 sequences of 300 bp upstream of every annotated translation start codon in the genome (translation start codons were used rather than putative transcription start sites as 5' UTRs are not well annotated in the genome). YMF motif finding was performed for motifs of length 6, 7, 8, 9 and $10 \mathrm{bp}$, allowing up to 2 ambiguous bases per motif. Each run produced 1000 predicted motifs ordered by Z-score, indicating overrepresentation of the motif against the background. The output of YMF includes many overlapping and therefore redundant motifs. To reduce this 
redundancy, the Find Explanators program was applied [80], which output $<20$ motifs for each length (6-10 bp). These motifs were clustered into subsets based on sequence similarity and/or non-random distribution in relation to the TSS. Non-random distribution of motifs in promoter regions was assessed using a Chi-square test of goodness of fit between the observed distribution and a uniform distribution across the promoter. Motifs defined using YMF/Find Explanators were verified by comparison with motifs identified using MEME [45,46], repeated 5 times for motif lengths of $6,7,8,9$ and $10 \mathrm{bp}$.

In order to assess the conservation of motifs between $A$. nidulans and $A$. fumigatus, putative upstream regions (300 bp upstream of the translation start codon ATG) were defined for all genes of both species. Motifs were identified in these regions by exact pattern matching using custom PERL scripts. A list of 5872 1:1 orthologue pairs was defined using search options in FungiDB [10]. All gene pairs in this list were scored as having the motif in both species, in A. nidulans only, in A. fumigatus only or in neither species. The significance of orthologues sharing the motif was tested using a chi-squared test.

\section{Mutant strain construction and analysis of meaB antisense transcription}

The meaBDintron mutant construct, in which the first intron had been excluded, was formed by fusion PCR [81] utilising a proofreading taq polymerase (KOD). The primers used for the first round of PCR were: meaB_F1 (5' -TCTTCGGCTAGTGTCCGAGT-3') with $\Delta$ intronRev (5' -CCGAGGTCGCATAGATAGGTTTCCTGCCA CCCTTCCG-3') and meaB_R4 (5'-TCGAGTGAGTG AGCATTTGG-3') with $\triangle$ intronFor (5' -GGGTGGCAGGAAACCTATCTATGCGACCTCGGAAGAG-3'). The initial products were combined and fused by PCR using primers meaB_F2 (5' -CTCGTAAAGGAGCTGGGTTG$3^{\prime}$ ) and meaB_R3 (5' -TGCAGGGATGGAGCTTTAGT$\left.3^{\prime}\right)$. This construct was then knocked in at the meaB locus by transformation into a $\triangle m e a B$ strain ( $\triangle$ meaB:AfpyrG, pyrG89, pabaB22 riboB2, $\triangle n k u A: \operatorname{argB}$ (argB2)) [41]. Transformants were selected for the loss of $A$ fpyrG, on the basis of resistance to 5-fluoroorotic acid and fidelity of the mutation was confirmed by PCR and Southern hybridisation. A transformant was then outcrossed and the strain used for mRNA assays had the genotype meaBDintron pantoB100. The $\triangle \mathrm{RNAi}$ strain used was RTMH.7 39 [43] which had the genotype $\Delta r r p B:: p y r G$; $\Delta \operatorname{rrpC}::$ metG $\Delta d c l B:: p y r G ; \Delta r s d A:: p y r G$. The areA49 strain, which has a loss of function mutation due to an 8-bp deletion beginning in are $A$ codon 75 [82], had the genotype are $A 49$ pabaA1.

Northern analysis was conducted using total RNA as described previously $[41,83]$. The meaB antisense was probed using the end labelled primer, meaB-ASprobe
(5'-GAACCATAATGACATGGCCATGGACCAGGTCG CCCCCAAGTC-3'). Quantification of meaB expression was conducted using a phosphorimager (STORM 860) and based on four independent replicates.

\section{Additional files}

Additional file 1: Additional study information. Additional text, tables and figures describing whole transcriptome and 5'-end alignments and analyses that were not included in the main text.

Additional file 2: Whole transcriptome read counts per locus. Raw and normalised (RPKM) read counts for all annotated loci ( $n=10827) .6$ loci (CADANIAG00010797, CADANIAG00010692, CADANIAG00010663, CADANIAG00010810, CADANIAG00010773, CADANIAG00010682) encoding spliceosomal RNAs and small nucleolar RNAs annotated as on the + strand were manually altered to the - strand based on depth of coverage across the loci.

Additional file 3: Transcripts predicted by whole transcriptome RNA-seq alignments. Text file (in '.gtf' format) containing the locations of putative transcripts predicted from alignment of whole transcriptome RNA-seq libraries of cells grown under different conditions. Instructions to view the data using the integrative genome browser (IGV) software are given in Additional file 1.

Additional file 4: Analysis of novel ncRNA and protein coding genes. Results of searches of novel transcripts against the Pfam and Rfam databases. Each ID in the column 'transcript_ID' represents a transcribed region predicted by the Cufflinks software, based on read coverage and its sequence and length are in columns 'transcript_sequence' and 'transcript_length(nt)'. 'ORF_ID' indicates the ID of an open reading frame present in the transcript, 'translated_ORF_sequence' is its amino acid sequence, 'proportion_of_transcript_ORF' indicates the proportion of the putative transcript that is ORF (a high value suggests a real protein coding gene). The following 15 columns descibe the results of a Pfam search, using the translated ORF as the query (see http://pfam.sanger.ac.uk/ for description). The next 15 columns describe the results of an Rfam search, using the putative transcript as the query (see http://rfam.sanger.ac.uk/ for description). The column 'Since annotated as..' records cases where the transcript was annotated in a later version of the annotation than the one analysed (red indicates no subsequent annotation).

Additional file 5: Introns. Text file (in '.bed' format) containing the locations of Introns identified by alignment of whole transcriptome RNAseq libraries of cells grown under different conditions. Instructions to view the data using the integrative genome browser (IGV) software are given in Additional file 1.

Additional file 6: Antisense transcription of annotated loci. Distribution of antisense reads across annotated loci. Raw read counts are shown for all annotated loci longer than $90 \mathrm{bp}(\mathrm{n}=10697) .5$ loci (CADANIAG00010797, CADANIAG00010663, CADANIAG00010810, CADANIAG00010773, CADANIAG00010682) encoding spliceosomal RNAs and small nucleolar RNAs annotated as on the + strand were manually altered to the - strand. Loci were scored $5^{\prime}$-biased if $>40 \%$ of reads mapped to the $5^{\prime}$ third and $<10 \%$ to the $3^{\prime}$ third, $3^{\prime}$-biased if $>40 \%$ of reads mapped to the $3^{\prime}$ third and $<10 \%$ to the $5^{\prime}$ third and middle-biased if $>75 \%$ of reads mapped to the central third. Note that raw read counts were to left, middle and right thirds irrespective of the orientation of the locus. Therefore, for a locus on the positive strand the left third is the $5^{\prime}$ end, while for a locus on the negative strand the left third is the $3^{\prime}$ end.

Additional file 7: Analysis of uncapped transcripts. Peaks of read head depth >100 in the 'no treatment' 5'-end library. The peaks should represent the 5' ends of uncapped transcripts. Many of the coverage peaks identify snoRNAs.

Additional file 8: Putative promoter regions. Text file (in '.gtf' format) containing the locations of putative promoter regions predicted from alignment of 5'-enriched RNA-seq libraries of cells grown under a single growth condition (nitrate as nitrogen source). Instructions to view the 
data using the integrative genome browser (IGV) software are given in Additional file 1.

Additional file 9: Putative promoter regions of annotated genes. Genomic locations of genes and their associated transcription start sites (TSS) and putative promoters. TSS are classified as 'tight', 'intermediate' and 'diffuse' by their confidence interval length (CIL) value, the length of the confidence interval around the major peak of read coverage representing the transcription start site $(<2 n t=' t i g h t$ ', $2 n t<C I L<4 n t=' i n t e r m e d i a t e '$, $>4 n t='$ 'diffuse').

Additional file 10: Gene ontology analysis of genes with different promoter types. Gene ontologies (GO) for processes significantly enriched in gene sets associated with 'tight', 'intermediate' and/or 'diffuse' transcription start sites, according to our classification. For each GO category, the 'cluster frequency' (of the particular gene set) and 'background frequency' (of all annotated genes in the genome) associated with that GO category are reported. The 'P-value', Bonferronicorrected for multiple testing, and 'FDR' (false discovery rate) are also reported, followed by colon-separated lists of the genes associated with the GO category and the GO IDs associated with the genes (which may be for the GO category itself, or a more specific sub-category of the broader GO category).

Additional file 11: Motifs that are significantly enriched (calculated by the YMF software) in promoters upstream of 'tight',

'intermediate' and 'diffuse' TSS. The table shows the motif, the number of occurrences of the motif in the set of promoter regions and the $Z$ score, measuring how much more common the motif is than a random sequence drawn from DNA with similar base frequencies. These are shown for each set of promoters associated with a type of TSS: 'tight', 'intermediate' and 'diffuse'. To distinguish real 'diffuse' TSS from stochastic noise (to enrich for real promoters), only those within 500 bp upstream of annotated genes were used.

\section{Abbreviations}

TSS: Transcription start site; NAT: Natural antisense transcript; CIL: Confidence interval length; RH: Read head; RT-PCR: Reverse transcription polymerase chain reaction; TAP: Tobacco acid pyrophosphatase; UTR: Untranslated region.

\section{Competing interests}

The authors declare that they have no competing interests.

\section{Authors' contributions}

Designed experiments: CS, IM, PW, GW, MXC. Conducted laboratory work: CS, PP, IM. Undertook bioinformatic analysis; CS, HW, GC, GW. Wrote manuscript: CS, GC, GW, MXC. All authors read and approved the final manuscript.

\section{Acknowledgements}

This work was supported by the BBSRC (BSR11014 and BB/G000573/1). The $\triangle$ RNAi strain used was provided by Nancy Keller. We are grateful to the support and collaboration of Jane Mabey Gilsenan (CADRE) and Jenifer Wortman and colleagues at AspGD for hosting these data on their respective genome browsers.

\section{Author details}

'Institute of Integrative Biology, University of Liverpool, Biosciences Building Crown Street, Liverpool L69 7ZB, UK. ²Department of Computer Science, University of Liverpool, Ashton Building, Ashton Street, Liverpool L69 3BX, UK. ${ }^{3}$ Department of Biomolecular and Sports Sciences, Faculty of Health and Life Sciences, Coventry University, James Starley Building, Coventry CV1 5FB, UK.

Received: 15 August 2013 Accepted: 15 November 2013 Published: 3 December 2013

\section{References}

1. Nierman WC, Pain A, Anderson MJ, Wortman JR, Kim HS, Arroyo J, Berriman M, Abe K, Archer DB, Bermejo C, Bennett J, Bowyer P, Chen D, Collins M, Coulsen R, Davies R, Dyer PS, Farman M, Fedorova N, Fedorova N, Feldblyum TV, Fischer R, Fosker N, Fraser A, García JL, García MJ, Goble A,
Goldman GH, Gomi K, Griffith-Jones S, et al: Genomic sequence of the pathogenic and allergenic filamentous fungus Aspergillus fumigatus. Nature 2005, 438:1151-1156.

2. Machida M, Asai K, Sano M, Tanaka T, Kumagai T, Terai G, Kusumoto K, Arima T, Akita O, Kashiwagi Y, Abe K, Gomi K, Horiuchi H, Kitamoto K, Kobayashi T, Takeuchi M, Denning DW, Galagan JE, Nierman WC, Yu J, Archer DB, Bennett JW, Bhatnagar D, Cleveland TE, Fedorova ND, Gotoh O, Horikawa H, Hosoyama A, Ichinomiya $M$, Igarashi $R$, et al: Genome sequencing and analysis of Aspergillus oryzae. Nature 2005, 438:1157-1161.

3. Pel HJ, de Winde JH, Archer DB, Dyer PS, Hofmann G, Schaap PJ, Turner G, de Vries RP, Albang R, Albermann K, Andersen MR, Bendtsen JD, Benen JA, van den Berg M, Breestraat S, Caddick MX, Contreras R, Cornell M, Coutinho PM, Danchin EG, Debets AJ, Dekker P, van Dijck PW, van Dijk A, Dijkhuizen L, Driessen AJ, d'Enfert C, Geysens S, Goosen C, Groot GS, et al: Genome sequencing and analysis of the versatile cell factory Aspergillus niger CBS 513.88. Nat Biotechnol 2007, 25:221-231.

4. Fedorova ND, Khaldi N, Joardar VS, Maiti R, Amedeo P, Anderson MJ, Crabtree J, Silva JC, Badger JH, Albarraq A, Angiuoli S, Bussey H, Bowyer P, Cotty PJ, Dyer PS, Egan A, Galens K, Fraser-Liggett CM, Haas BJ, Inman JM, Kent R, Lemieux S, Malavazi I, Orvis J, Roemer T, Ronning CM, Sundaram JP, Sutton G, Turner G, Venter JC, et al: Genomic islands in the pathogenic filamentous fungus Aspergillus fumigatus. PLoS Genet 2008, 4:e1000046.

5. Andersen MR, Salazar MP, Schaap PJ, van de Vondervoort PJ, Culley D, Thykaer J, Frisvad JC, Nielsen KF, Albang R, Albermann K, Berka RM, Braus GH, BrausStromeyer SA, Corrochano LM, Dai Z, van Dijck PW, Hofmann G, Lasure LL, Magnuson JK, Menke H, Meijer M, Meijer SL, Nielsen JB, Nielsen ML, van Ooyen AJ, Pel HJ, Poulsen L, Samson RA, Stam H, Tsang A, et al: Comparative genomics of citric-acid-producing Aspergillus niger ATCC 1015 versus enzyme-producing CBS 513.88. Genome Res 2011, 21:885-897.

6. Galagan JE, Calvo SE, Cuomo C, Ma LJ, Wortman JR, Batzoglou S, Lee SI, Baştürkmen M, Spevak CC, Clutterbuck J, Kapitonov V, Jurka J, Scazzocchio C, Farman M, Butler J, Purcell S, Harris S, Braus GH, Draht O, Busch S, D'Enfert C, Bouchier C, Goldman GH, Bell-Pedersen D, Griffiths-Jones S, Doonan JH, Yu J, Vienken K, Pain A, Freitag M, et al: Sequencing of Aspergillus nidulans and comparative analysis with $A$. fumigatus and A. oryzae. Nature 2005, 438:1105-1115.

7. AspGD. http://www.aspgd.org/

8. CADRE. http://www.cadre-genomes.org.uk/.

9. Ensemb/ Genomes ftp site. ftp://ftp.ensemblgenomes.org/pub/fungi/.

10. FungiDB web resource. http://fungidb.org/fungidb/.

11. Wortman JR, Gilsenan JM, Joardar V, Deegan J, Clutterbuck J, Andersen MR, Archer D, Bencina M, Braus G, Coutinho P, von Döhren H, Doonan J, Driessen AJ, Durek P, Espeso E, Fekete E, Flipphi M, Estrada CG, Geysens S, Goldman G, de Groot PW, Hansen K, Harris SD, Heinekamp T, Helmstaedt K, Henrissat B, Hofmann G, Homan T, Horio T, Horiuchi H, et al: The 2008 update of the Aspergillus nidulans genome annotation: a community effort. Fungal Genet Biol 2009, 46(Suppl 1):2-13.

12. Wang Z, Gerstein M, Snyder M: RNA-Seq: a revolutionary tool for transcriptomics. Nat Rev Genet 2009, 10:57-63.

13. Katayama S, Tomaru Y, Kasukawa T, Waki K, Nakanishi M, Nakamura M, Nishida H, Yap CC, Suzuki M, Kawai J, Suzuki H, Carninci P, Hayashizaki Y, Wells C, Frith M, Ravasi T, Pang KC, Hallinan J, Mattick J, Hume DA, Lipovich L, Batalov S, Engström PG, Mizuno Y, Faghihi MA, Sandelin A, Chalk AM, Mottagui-Tabar S, Liang Z, Lenhard B, et al: Antisense transcription in the mammalian transcriptome. Science 2005, 309:1564-1566.

14. Faghihi MA, Wahlestedt C: Regulatory roles of natural antisense transcripts. Nat Rev Mol Cell Biol 2009, 10:637-643.

15. Gibbons JG, Beauvais A, Beau R, McGary KL, Latgé JP, Rokas A: Global transcriptome changes underlying colony growth in the opportunistic human pathogen Aspergillus fumigatus. Eukaryot Cell 2012, 11:68-78.

16. Delmas S, Pullan ST, Gaddipati S, Kokolski M, Malla S, Blythe MJ, Ibbett R, Campbell M, Liddell S, Aboobaker A, Tucker GA, Archer DB: Uncovering the genome-wide transcriptional responses of the filamentous fungus Aspergillus niger to lignocellulose using RNA sequencing. PLoS Genet 2012, 8:e1002875.

17. van Leeuwen MR, Krijgsheld P, Bleichrodt R, Menke H, Stam H, Stark J, Wösten HA, Dijksterhuis J: Germination of conidia of Aspergillus niger is accompanied by major changes in RNA profiles. Stud Mycol 2013, 74:59-70.

18. Novodvorska M, Hayer K, Pullan ST, Wilson R, Blythe MJ, Stam H, Stratford M, Archer DB: Trancriptional landscape of Aspergillus niger at breaking of conidial dormancy revealed by RNA-sequencing. BMC Genomics 2013, $14: 246$. 
19. Yu J, Fedorova ND, Montalbano BG, Bhatnagar D, Cleveland TE, Bennett JW, Nierman WC: Tight control of mycotoxin biosynthesis gene expression in Aspergillus flavus by temperature as revealed by RNA-Seq. FEMS Microbiol Lett 2011, 322:145-149.

20. Lin JQ, Zhao XX, Zhi QQ, Zhao M, He ZM: Transcriptomic profiling of Aspergillus flavus in response to 5-azacytidine. Fungal Genet Biol 2013, 56:78-86.

21. Wang B, Guo G, Wang C, Lin Y, Wang X, Zhao M, Guo Y, He M, Zhang Y, Pan L: Survey of the transcriptome of Aspergillus oryzae via massively parallel mRNA sequencing. Nucleic Acids Res 2010, 38:5075-5087.

22. Rokas A, Gibbons JG, Zhou X, Beauvais A, Latgé JP: The diverse applications of RNA-seq for functional genomic studies in Aspergillus fumigatus. Ann N Y Acad Sci 2012, 1273:25-34

23. Wery M, Descrimes $M$, Thermes $C$, Gautheret D, Morillon A: Zinc-mediated RNA fragmentation allows robust transcript reassembly upon whole transcriptome RNA-Seq. Methods. in press.

24. Shiraki T, Kondo S, Katayama S, Waki K, Kasukawa T, Kawaji H, Kodzius R, Watahiki A, Nakamura M, Arakawa T, Fukuda S, Sasaki D, Podhajska A, Harbers M, Kawai J, Carninci P, Hayashizaki Y: Cap analysis gene expression for highthroughput analysis of transcriptional starting point and identification of promoter usage. Proc Natl Acad Sci U S A 2003, 100:15776-15781.

25. Hashimoto S, Suzuki Y, Kasai Y, Morohoshi K, Yamada T, Sese J, Morishita S, Sugano S, Matsushima K: 5 '-end SAGE for the analysis of transcriptional start sites. Nat Biotechnol 2004, 22:1146-1149.

26. Gowda M, Li H, Wang GL: Robust analysis of 5'-transcript ends: a highthroughput protocol for characterization of sequence diversity of transcription start sites. Nat Protoc 2007, 2:1622-1632.

27. de Hoon M, Hayashizaki Y: Deep cap analysis gene expression (CAGE): genome-wide identification of promoters, quantification of their expression, and network inference. Biotechniques 2008, 44:627-632.

28. Pontecorvo G, Roper JA, Hemmons LM, MacDonald KD, Bufton AWJ: The genetics of Aspergillus nidulans. Adv Genet 1953, 5:141-238.

29. Burge SW, Daub J, Eberhardt R, Tate J, Barquist L, Nawrocki EP, Eddy SR, Gardner PP, Bateman A: Rfam 11.0: 10 years of RNA families. Nucleic Acids Res 2013, 41(Database issue):D226-D232

30. Punta M, Coggill PC, Eberhardt RY, Mistry J, Tate J, Boursnell C, Pang N, Forslund K, Ceric G, Clements J, Heger A, Holm L, Sonnhammer ELL, Eddy SR, Bateman A, Finn RD: The Pfam protein families database. Nucleic Acids Res 2012, 40(Database Issue):D290-D301.

31. Borsuk P, Przykorska A, Blachnio K, Koper M, Pawlowicz JM, Pekala M, Weglenski $P$ : L-arginine influences the structure and function of arginase mRNA in Aspergillus nidulans. Biol Chem 2007, 388:135-144.

32. Chang KY, Georgianna DR, Heber S, Payne GA, Muddiman DC: Detection of alternative splice variants at the proteome level in Aspergillus flavus. J Proteome Res 2010, 9:1209-1217.

33. Chang KY, Muddiman DC: Identification of alternative splice variants in Aspergillus flavus through comparison of multiple tandem MS search algorithms. BMC Genomics 2011, 12:358.

34. Trevisan GL: Transcription of Aspergillus nidulans pacC is modulated by alternative RNA splicing of palB. FEBS Lett 2011, 585:3442-3445.

35. Freitag J, Ast J, Bölker M: Cryptic peroxisomal targeting via alternative splicing and stop codon read-through in fungi. Nature 2012, 485:522-525.

36. Liu N, Xiao ZD, Yu CH, Shao P, Liang YT, Guan DG, Yang JH, Chen CL, Qu LH, Zhou H: SnoRNAs from the filamentous fungus Neurospora crassa: structural, functional and evolutionary insights. BMC Genomics 2009, 10:515.

37. Ponting $\mathrm{CP}$, Oliver $\mathrm{PL}$, Reik W: Evolution and functions of long noncoding RNAs. Cell 2009, 136:629-641.

38. Smith CA, Robertson D, Yates B, Nielsen DM, Brown D, Dean RA, Payne GA The effect of temperature on Natural Antisense Transcript (NAT) expression in Aspergillus flavus. Curr Genet 2008, 54:241-269.

39. David L, Huber W, Granovskaia M, Toedling J, Palm CJ, Bofkin L, Jones T, Davis RW, Steinmetz LM: A high-resolution map of transcription in the yeast genome. Proc Natl Acad Sci U S A 2006, 103:5320-5325.

40. Polley SD, Caddick MX: Molecular characterisation of meaB, a novel gene affecting nitrogen metabolite repression in Aspergillus nidulans. FEBS Lett 1996, 388:200-205.

41. Wagner D, Schmeinck A, Mos M, Morozov IY, Caddick MX, Tudzynski B: The bZIP transcription factor MeaB mediates nitrogen metabolite repression at specific loci. Eukaryot Cell 2010, 9:1588-1601.

42. Caddick MX, Arst HN Jr, Taylor LH, Johnson RI, Brownlee AG: Cloning of the regulatory gene are $A$ mediating nitrogen metabolite repression in Aspergillus nidulans. EMBO J 1986, 5:1087-1090.
43. Hammond TM, Bok JW, Andrewski MD, Reyes-Domínguez Y, Scazzocchio C, Keller NP: RNA silencing gene truncation in the filamentous fungus Aspergillus nidulans. Eukaryot Cell 2008, 7:339-349.

44. Sinha S, Tompa M: YMF: A program for discovery of novel transcription factor binding sites by statistical overrepresentation. Nucleic Acids Res 2003, 31:3586-3588.

45. Bailey TL, Elkan C: Fitting a mixture model by expectation maximization to discover motifs in biopolymers. In Proceedings of the Second International Conference on Intelligent Systems for Molecular Biology: 14-17 August 1994; Stanford, California. Edited by Russ A, Douglas B, Peter K, Richard L, David S. Menlo Park, California: AAAI Press; 1994:28-36.

46. Bailey TL, Boden M, Buske FA, Frith M, Grant CE, Clementi L, Ren J, Li WW, Noble WS: MEME SUITE: tools for motif discovery and searching. Nucleic Acids Res 2009, 37(Web Server issue):W202-W208.

47. Yamamoto A, Mizukami $Y$, Sakurai H: Identification of a novel class of target genes and a novel type of binding sequence of heat shock transcription factor in Saccharomyces cerevisiae. J Biol Chem 2005, 280:11911-11919.

48. Kulmburg P, Mathieu M, Dowzer C, Kelly J, Felenbok B: Specific binding sites in the alcR and alcA promoters of the ethanol regulon for the CREA repressor mediating carbon catabolite repression in Aspergillus nidulans. Mol Microbiol 1993, 7:847-857.

49. Ravagnani A, Gorfinkiel L, Langdon T, Diallinas G, Adjadj E, Demais S, Gorton D, Arst HN Jr, Scazzocchio C: Subtle hydrophobic interactions between the seventh residue of the zinc finger loop and the first base of an HGATAR sequence determine promoter-specific recognition by the Aspergillus nidulans GATA factor AreA. EMBO J 1997, 16:3974-3986.

50. Skromne I, Sánchez O, Aguirre J: Starvation stress modulates the expression of the Aspergillus nidulans brlA regulatory gene. Microbiology 1995, 141:21-28.

51. Garzia A, Etxebeste O, Rodríguez-Romero J, Fischer R, Espeso EA, Ugalde U: Transcriptional changes in the transition from vegetative cells to asexual development in the model fungus Aspergillus nidulans. Eukaryot Cell 2013, 12:311-321.

52. Kapranov P, Cawley SE, Drenkow J, Bekiranov S, Strausberg RL, Fodor SP, Gingeras TR: Large-scale transcriptional activity in chromosomes 21 and 22. Science 2002, 296:916-919.

53. Carninci P, Kasukawa T, Katayama S, Gough J, Frith MC, Maeda N, Oyama R, Ravasi T, Lenhard B, Wells C, Kodzius R, Shimokawa K, Bajic VB, Brenner SE, Batalov S, Forrest AR, Zavolan M, Davis MJ, Wilming LG, Aidinis V, Allen JE, Ambesi-Impiombato A, Apweiler R, Aturaliya RN, Bailey TL, Bansal M, Baxter $\mathrm{L}$, Beisel KW, Bersano T, Bono $\mathrm{H}$, et al: The transcriptional landscape of the mammalian genome. Science 2005, 309:1559-1563.

54. Kapranov P, Cheng J, Dike S, Nix DA, Duttagupta R, Willingham AT, Stadler PF, Hertel J, Hackermüller J, Hofacker IL, Bell I, Cheung E, Drenkow J, Dumais E, Patel S, Helt G, Ganesh M, Ghosh S, Piccolboni A, Sementchenko V, Tammana $\mathrm{H}$, Gingeras TR: RNA maps reveal new RNA classes and a possible function for pervasive transcription. Science 2007, 316:1484-1488.

55. Hangauer MJ, Vaughn IW, McManus MT: Pervasive Transcription of the Human Genome Produces Thousands of Previously Unidentified Long Intergenic Noncoding RNAs. PLoS Genet 2013, 9:e1003569.

56. Kervestin $\mathrm{S}$, Jacobson A: NMD: a multifaceted response to premature translational termination. Nat Rev Mol Cell Biol 2012, 13:700-712.

57. Kelly SM, Corbett AH: Messenger RNA export from the nucleus: a series of molecular wardrobe changes. Traffic 2009, 10:1199-1208.

58. Yassour M, Pfiffner J, Levin JZ, Adiconis X, Gnirke A, Nusbaum C, Thompson DA, Friedman N, Regev A: Strand-specific RNA sequencing reveals extensive regulated long antisense transcripts that are conserved across yeast species. Genome Biol 2010, 11:R87

59. Goodman AJ, Daugharthy ER, Kim J: Pervasive antisense transcription is evolutionarily conserved in budding yeast. Mol Biol Evol 2013, 30:409-421.

60. Bautista LF, Aleksenko A, Hentzer M, Santerre-Henriksen A, Nielsen J: Antisense silencing of the creA gene in Aspergillus nidulans. Appl Environ Microbiol 2000, 66:4579-4581.

61. Suzuki Y, Taira H, Tsunoda T, Mizushima-Sugano J, Sese J, Hata H, Ota T, Isogai T, Tanaka T, Morishita S, Okubo K, Sakaki Y, Nakamura Y, Suyama A, Sugano S: Diverse transcriptional initiation revealed by fine, large-scale mapping of mRNA start sites. EMBO Rep 2001, 2:388-393.

62. Carninci P, Sandelin A, Lenhard B, Katayama S, Shimokawa K, Ponjavic J, Semple CA, Taylor MS, Engström PG, Frith MC, Forrest AR, Alkema WB, Tan SL, Plessy C, Kodzius R, Ravasi T, Kasukawa T, Fukuda S, Kanamori-Katayama 
M, Kitazume Y, Kawaji H, Kai C, Nakamura M, Konno H, Nakano K, MottaguiTabar S, Arner P, Chesi A, Gustincich S, Persichetti F, et al: Genome-wide analysis of mammalian promoter architecture and evolution. Nat Genet 2006, 38:626-635.

63. Sandelin A, Carninci P, Lenhard B, Ponjavic J, Hayashizaki Y, Hume DA: Mammalian RNA polymerase II core promoters: insights from genome-wide studies. Nat Rev Genet 2007, 8:424-436.

64. Cheng CK, Au CH, Wilke SK, Stajich JE, Zolan ME, Pukkila PJ, Kwan HS: 5'-Serial Analysis of Gene Expression studies reveal a transcriptomic switch during fruiting body development in Coprinopsis cinerea. BMC Genomics 2013, 14:195.

65. Waern K, Snyder M: Extensive transcript diversity and novel upstream open reading frame regulation in yeast. G3 2013, 3:343-352.

66. Suzuki Y, Tsunoda T, Sese J, Taira H, Mizushima-Sugano J, Hata H, Ota T, Isogai T, Tanaka T, Nakamura Y, Suyama A, Sakaki Y, Morishita S, Okubo K, Sugano S: Identification and characterization of the potential promoter regions of 1031 kinds of human genes. Genome Res 2001, 11:677-684.

67. Hoskins RA, Landolin JM, Brown JB, Sandler JE, Takahashi H, Lassmann T, Yu C, Booth BW, Zhang D, Wan KH, Yang L, Boley N, Andrews J, Kaufman TC, Graveley BR, Bickel PJ, Carninci P, Carlson JW, Celniker SE: Genome-wide analysis of promoter architecture in Drosophila melanogaster. Genome Res 2011, 21:182-192.

68. Yamamoto YY, Yoshioka Y, Hyakumachi M, Obokata J: Characteristics of core promoter types with respect to gene structure and expression in Arabidopsis thaliana. DNA Res 2011, 18:333-342.

69. Ballance DJ: Sequences important for gene-expression in filamentous fungi. Yeast 1986, 2:229-236.

70. Ohler U, Liao GC, Niemann H, Rubin GM: Computational analysis of core promoters in the Drosophila genome. Genome Biol 2002, 3:RESEARCH0087.

71. Cove DJ: The induction and repression of nitrate reductase in the fungus Aspergillus nidulans. Biochimica et biophysica acta 1966, 113:1-56.

72. Sokolovsky V, Kaldenhoff R, Ricci M, Russo VEA: Fast and reliable mini-prep RNA extraction from Neurospora crassa. Fungal Genetics Newsletter 1990, 37:41-42.

73. Langmead B, Trapnell C, Pop M, Salzberg SL: Ultrafast and memory-efficient alignment of short DNA sequences to the human genome. Genome Biol 2009, 10:R25

74. Trapnell C, Pachter L, Salzberg SL: TopHat: discovering splice junctions with RNA-Seq. Bioinformatics 2009, 25:1105-1111.

75. Mabey Gilsenan J, Cooley J, Bowyer P: CADRE: the Central Aspergillus Data REpository 2012. Nucleic Acids Res 2012, 40(Database issue):D660-D666.

76. Arnaud MB, Chibucos MC, Costanzo MC, Crabtree J, Inglis DO, Lotia A, Orvis J, Shah P, Skrzypek MS, Binkley G, Miyasato SR, Wortman JR, Sherlock G: The Aspergillus Genome Database, a curated comparative genomics resource for gene, protein and sequence information for the Aspergillus research community. Nucleic Acids Res 2010, 38(Database issue):D420-D427.

77. Trapnell C, Williams BA, Pertea G, Mortazavi A, Kwan G, van Baren MJ, Salzberg SL, Wold BJ, Pachter L: Transcript assembly and quantification by RNA-Seq reveals unannotated transcripts and isoform switching during cell differentiation. Nat Biotechnol 2010, 28:511-515.

78. Rice P, Longden I, Bleasby A: EMBOSS: the European Molecular Biology Open Software Suite. Trends Genet 2000, 16:276-277.

79. Pfam web server. http://pfam.sanger.ac.uk/.

80. Blanchette $M$, Sinha S: Separating real motifs from their artifacts. Bioinformatics 2001, 17(Suppl):S30-S38.

81. Yu JH, Hamari Z, Han KH, Seo JA, Reyes-Domínguez Y, Scazzocchio C: Double-joint PCR: a PCR-based molecular tool for gene manipulations in filamentous fungi. Fungal Genet Biol 2004, 41:973-981.

82. Caddick MX, Arst HN Jr: Deletion of the $389 \mathrm{~N}$-terminal residues of the transcriptional activator AREA does not result in nitrogen metabolite derepression in Aspergillus nidulans. J Bacteriol 1998, 180:5762-5764.

83. Platt A, Langdon T, Arst HN Jr, Kirk D, Tollervey D, Sanchez JM, Caddick MX: Nitrogen metabolite signalling involves the C-terminus and the GATA domain of the Aspergillus transcription factor AREA and the $3^{\prime}$ untranslated region of its mRNA. EMBO J 1996, 15:2791-2801.

doi:10.1186/1471-2164-14-847

Cite this article as: Sibthorp et al:: Transcriptome analysis of the filamentous fungus Aspergillus nidulans directed to the global identification of promoters. BMC Genomics 2013 14:847.

\section{Submit your next manuscript to BioMed Central and take full advantage of:}

- Convenient online submission

- Thorough peer review

- No space constraints or color figure charges

- Immediate publication on acceptance

- Inclusion in PubMed, CAS, Scopus and Google Scholar

- Research which is freely available for redistribution 